\title{
SUBGROUP CONDITIONS FOR GROUPS ACTING FREELY ON PRODUCTS OF SPHERES
}

\author{
JUDITH H. SILVERMAN
}

\begin{abstract}
Let $d$ and $h$ be integers such that either $d \geq 2$ and $h=2^{d}-1$, or $d=4$ and $h=5$. Suppose that the group $\mathscr{G}$ contains an elementary-abelian 2-subgroup $E_{d}$ of rank $d$ with an element $\sigma$ of order $h$ in its normalizer. We show that if $\mathscr{G}$ admits a free and $\mathbf{F}_{2}$-cohomologically trivial action on $\left(S^{n}\right)^{d}$, then some nontrivial power of $\sigma$ centralizes $E_{d}$.

The cohomology ring $H^{*}\left(E_{d} ; \mathbf{F}_{2}\right) \simeq \mathbf{F}_{2}\left[y_{1}, \ldots, y_{d}\right]$ is a module over the Steenrod algebra $\mathscr{A}(2)$. Let $\theta \in \mathbf{F}_{2}\left[y_{1}, \ldots, y_{d}\right]$, and let $c \geq d-2$ be an integer. We show that $\theta$ divides $S q^{2^{i}}(\theta)$ in the polynomial ring for $0 \leq i \leq$ $c \Leftrightarrow \theta=\tau^{2^{c-d+3}} \pi$, where $\tau$ divides $S q^{2^{i}}(\tau)$ for $0 \leq i \leq d-3$ and $\pi$ is a product of linear forms.
\end{abstract}

\section{Part I. Preliminaries}

\section{INTRODUCTION}

Suppose that the group $\mathscr{G}$ acts freely and $\mathbf{F}_{2}$-cohomologically trivially on a finite complex $X \sim_{2}\left(S^{n}\right)^{d}$ (that is, $X$ has the mod-2 cohomology of the Cartesian product $\left.\left(S^{n}\right)^{d}\right)$. A theorem due to Carlsson [Car 80] states that all elementary-abelian 2-subgroups of $\mathscr{G}$ have rank $\leq d$. The purpose of this paper is to find constraints on the normalizers of any rank- $d$ elementary-abelian 2-subgroups $E_{d}$ of $\mathscr{G}$. Note that if $\sigma$ is an element of the normalizer $N_{G}\left(E_{d}\right)$, then the group generated by $\sigma$ and $E_{d}$ is a semidirect product $\langle\sigma\rangle \ltimes_{\tau} E_{d}$ over some homomorphism $\tau:\langle\sigma\rangle \rightarrow \operatorname{Aut}\left(E_{d}\right) ; E_{d}$ sits inside this group as a normal subgroup. Since $E_{d} \simeq\left(\mathbf{F}_{2}\right)^{d}$ as abelian groups, one may think of $\tau$ as a degree- $d$ representation of $\langle\sigma\rangle$ over $\mathbf{F}_{2}$; if no nontrivial power of $\sigma$ is in the centralizer of $E_{d}$, then the representation $\tau$ is faithful. We have the following:

Theorems III.5 and III.11 (Main Theorem). Let $d$ and $h$ be integers such that either $d \geq 2$ and $h=2^{d}-1$, or $d=4$ and $h=5$. Suppose that the group $\mathscr{G}$ contains a semidirect product $G \simeq \mathbf{Z} /(h) \ltimes_{\tau} E_{d}$, where $E_{d}$ is an elementaryabelian 2-group and $\tau$ is a faithful irreducible degree-d representation of $\mathbf{Z} /(h)$ over $\mathbf{F}_{2}$. Then $\mathscr{G}$ does not admit free, $\mathbf{F}_{2}$-cohomologically trivial actions on any finite complex $X \sim_{2}\left(S^{n}\right)^{d}$ for any value of $n$ (if $(d, h)=(4,5)$, one requires that $n$ not be of the form $\left.2^{l} \cdot 5-1\right)$.

Received by the editors September 28, 1990.

1980 Mathematics Subject Classification (1985 Revision). Primary 55M35, 55S10.

Key words and phrases. Steenrod algebra, free group actions. 
If $(d, h)=(2,3)$ or $(3,7)$, then in fact $\mathscr{G}$ admits no such actions on any $X \sim_{2}\left(S^{n}\right)^{l}$ for any $l \geq d$; see [Oli78 and Car81] for simple proofs of these theorems for the case $(d, h)=(2,3)$ and the cases $(d, h)=(2,3)$ and $(d, h)=(3,7)$, respectively.

I thank Gunnar Carlsson for suggesting this question and for many helpful conversations. I am also grateful to the referee for pointing out that the groups $\mathbf{Z} /\left(2^{d}-1\right) \ltimes_{\tau} E_{d}$ occur in the finite simple groups $P S L_{2}\left(\mathbf{F}_{2^{d}}\right)$ as normalizers of the 2-Sylow subgroup, and consequently that there are an infinite number of simple groups of rank $d$ not acting freely on $X \sim_{2}\left(S^{n}\right)^{d}$.

\section{Notation}

From now on, we write $k$ for the field of two elements $\mathbf{F}_{2}$. Let $L \supseteq K \supseteq k$ be field extensions, and let $\mathscr{C}=L\left[z_{1}, \ldots, z_{d}\right]$ be a graded polynomial algebra over $L$ on generators of degree 1 . We denote the module of homogeneous elements of degree $m$ by $\mathscr{C}_{(m)}$. Henceforth, all polynomials under discussion will be assumed to be homogeneous. The degree of a homogeneous polynomial $\phi$ is denoted by $\operatorname{deg}(\phi)$, and its degree as a polynomial in the single variable $z_{i}$ by $\operatorname{deg}_{i}(\phi)$. Let $\operatorname{Mon}(\mathscr{C}) \subset \mathscr{C}$ be the set of monomials of $\mathscr{C}$ with coefficient 1 . Any polynomial $0 \neq \phi \in \mathscr{C}$ is uniquely a sum $\phi=\sum\left\{f_{\mu} \mu \mid \mu \in \operatorname{Mon}(\phi)\right\}$ where $0 \neq f_{\mu} \in L$ and $\operatorname{Mon}(\phi) \subset \operatorname{Mon}(\mathscr{C})$. The elements of $\operatorname{Mon}(\phi)$ are the monomials of $\phi$. We write $\partial / \partial z_{i}$ for the familiar derivations of $L\left[z_{1}, \ldots, z_{t}\right]$. Since $\operatorname{char}(L)=2$, we have the following useful characterizations of the squares of $L\left[z_{1}, \ldots, z_{t}\right]$ :

$$
\begin{aligned}
& \phi \in \mathscr{C} \text { is a square } \Leftrightarrow \partial(\phi) / \partial z_{i}=0 \\
& \qquad f o r 1 \leq i \leq d \Leftrightarrow \mu \text { is a square for all } \mu \in \operatorname{Mon}(\phi) .
\end{aligned}
$$

Let $\mathscr{R}$ be a graded $K$-subalgebra of $\mathscr{C}$ and $\operatorname{Mat}_{n}(\mathscr{R})$ the ring of $(n \times n)$ matrices over $\mathscr{R}$. We denote the determinant of a matrix $\mathscr{A} \in \operatorname{Mat}_{n}(\mathscr{R})$ by $\delta(\mathscr{A})$. A matrix $\mathscr{A} \in \operatorname{Mat}_{n}(\mathscr{R})$ is diagonalizable over $k$ if the matrix $\mathscr{E}^{-1} \mathscr{A} \mathscr{E}$ is diagonal for some invertible matrix $\mathscr{E} \in \operatorname{Mat}_{n}(k)$, or equivalently, if $k^{n} \subset \mathscr{R}^{n}$ has a $k$-basis consisting of eigenvectors of $\mathscr{A}$ viewed as a linear transformation on $\mathscr{R}^{n}$.

It is also convenient to introduce the following notation:

Notation. Let $\left\{\phi_{1}, \ldots, \phi_{s}\right\}$ be polynomials of $\mathscr{R}$. Then $\mathscr{I}\left\{\phi_{1}, \ldots, \phi_{s}\right\}$ is the $\mathscr{R}$-ideal generated by the $\phi_{i}, \mathscr{A}\left\{\phi_{1}, \ldots, \phi_{s}\right\}$ is the subalgebra they generate, and $\mathscr{V}\left\{\phi_{1}, \ldots, \phi_{s}\right\} \subset \mathscr{R}$ is the $K$-vector subspace they span.

Definition I.1. A set of (homogeneous) elements $\mathscr{S}=\left\{\phi_{1}, \ldots, \phi_{s}\right\} \subset \mathscr{R}_{\geq 1}$ is an independent set of $s$ elements if for each $i, \phi_{i}$ is not a 0 -divisor in $\mathscr{R} / \mathscr{I}_{i-1}$, where $\mathscr{I}_{0}=0$ and $\mathscr{I}_{j}=\mathscr{I}\left\{\phi_{1}, \ldots, \phi_{j-1}\right\}$ for $1 \leq j \leq s-1$. If $\operatorname{deg}\left(\phi_{1}\right)=\operatorname{deg}\left(\phi_{2}\right)=\cdots=\operatorname{deg}\left(\phi_{s}\right)=m$, then $\mathscr{S}$ is a level independent set of degree $m$.

It is proven in [Ser65a], where independent sets are referred to as $\mathscr{R}$-suites, that the above condition is independent of the order of the $\phi_{i}$ 's. In particular, if $\rho \phi_{i} \in \mathscr{I}\left\{\phi_{j} \mid j \neq i\right\}$ for some $\rho \in \mathscr{R}$, then in fact the coefficient $\rho \in$ $\mathcal{I}\left\{\phi_{j} \mid j \neq i\right\}$. The following consequence will be used repeatedly:

Fact 1.2. 1. Let $\mathscr{S}=\left\{\phi_{1}, \ldots, \phi_{s}\right\} \subset \mathscr{R}$ be an independent set, and suppose that $\sum_{i=1}^{s} \alpha_{i} \phi_{i}=0$ for some $\alpha_{1}, \ldots, \alpha_{s} \in \mathscr{R}$. If for some $i$ we have $\operatorname{deg}\left(\alpha_{i}\right)<$ $\operatorname{deg}\left(\phi_{j}\right)$ for all $j \neq i$, then $\alpha_{i}=0$. 
2. Let $\mathscr{T}=\left\{\psi_{1}, \ldots, \psi_{s}\right\} \subset \mathscr{R}$ be a level independent set of degree $m$, and suppose that $\mathscr{M} \in \operatorname{Mat}\left(\mathscr{R}_{(n)}\right)$ is a matrix for which $\mathscr{M}\left(\psi_{1}, \ldots, \psi_{s}\right)^{T}=0$ or $\left(\psi_{1}, \ldots, \psi_{s}\right) \mathscr{M}=0$. Then either $\mathscr{M}=0$ or $n \geq m$. In particular, if $\sum_{i=1}^{s} \beta_{i} \psi_{i}=0$ for some $\beta_{1}, \ldots, \beta_{s} \in \mathscr{R}$ of degree $n$, then either $n \geq m$ or $\beta_{i}=0$ for all $i$.

Definition I.3. We say a set of (homogeneous) elements $\mathscr{S}=\left\{\phi_{1}, \ldots, \phi_{s}\right\} \subset$ $\mathscr{R}$ is an s-element homogeneous system of parameters (h.s.o.p.) if $\mathscr{R}$ is a finitely-generated module over $\mathscr{A}\left\{\phi_{1}, \ldots, \phi_{s}\right\}$. If $\operatorname{deg}\left(\phi_{1}\right)=\operatorname{deg}\left(\phi_{2}\right)=\cdots=$ $\operatorname{deg}\left(\phi_{s}\right)=m$, we say $\mathscr{S}$ is a level system of parameters (l.s.o.p.) of degree $m$.

From [Ser65a], we have

Proposition I.4. Let $\mathscr{R} \subset \mathscr{C}=L\left[z_{1}, \ldots, z_{d}\right]$ be a $K$-subalgebra such that $\mathscr{C}$ is finitely generated as an $\mathscr{R}$-module. Then

1. no independent set of $\mathscr{R}$ has more than d elements,

2. for $1 \leq s \leq d$, any s-element independent set (resp. h.s.o.p.) of $\mathscr{R}$ is an s-element independent set (resp. h.s.o.p.) of $\mathscr{C}$, and

3. the d-element independent sets of $\mathscr{R}$ are precisely the d-element h.s.o.p.'s of $\mathscr{R}$.

\section{COHOMOLOGY OF A TWISTED PRODUCT}

Let $\mathscr{G}$ be a group, $E_{d} \subset \mathscr{G}$ an elementary-abelian 2-subgroup of rank $d$, and $N_{\mathscr{G}}\left(E_{d}\right)$ the normalizer of $E_{d}$ in $\mathscr{G}$, acting on $E_{d}$ by conjugation. Suppose that $N_{\mathscr{G}}\left(E_{d}\right)$ contains an element $\eta$ of odd order $h$, no nontrivial power of which acts trivially on $E_{d}$. Let $H=\langle\eta\rangle$, and define $G$ to be $\left\langle\eta, E_{d}\right\rangle$, the group generated by $\eta$ and $E_{d}$.

Notation. We say $G=\left\langle\eta, E_{d}\right\rangle$ is of type I if $d \geq 2$ and $h=2^{d}-1$, and of type II if $d=4$ and $h=5$.

The main theorem states that if $G$ is of type I (resp. II), then the larger group $\mathscr{G}$ admits no free, $k$-cohomologically trivial action on any space $X \sim_{2}\left(S^{n}\right)^{d}$, for any $n$ (resp. for any $n$ not of the form $2^{l} \cdot 5-1$ ). The method of proof is to show that even the subgroup $G$ admits no such actions. In fact, $\mathscr{G}$ itself drops out of the picture and all computations take place within the cohomology of the subgroup $G$. Much of the discussion holds for all groups of the form $\left\langle\eta, E_{d}\right\rangle$, with no restrictions on $d$ and $h$ except those implicit in the condition on $\eta$. We will only restrict to groups of types I and II where necessary.

Following the approach of [Car81], we consider the cohomology algebra $H^{*}(G)$ of $G$, defined to be the cohomology (with $k$-coefficients) of the classifying space $B G$ of $G$. As mentioned above, the group $G$ may be regarded as a semidirect product $H \ltimes_{\tau} E_{d}$ for a suitable irreducible faithful representation $\tau: H \rightarrow G l_{d}(k)$. There is a well-known isomorphism of graded $k$-algebras

$$
H^{*}\left(E_{d}\right) \simeq k\left[y_{1}, \ldots, y_{d}\right],
$$

where $k\left[y_{1}, \ldots, y_{d}\right]$ is the polynomial algebra on $d$ generators $y_{1}, \ldots, y_{d}$ of degree 1 . The representation $\tau$ induces an action of $H$ on $k\left[y_{1}, \ldots, y_{d}\right]$, determined by multiplicity by its restriction $\rho$ to $H^{1}\left(E_{d}\right) \simeq k\left[y_{1}, \ldots, y_{d}\right]_{(1)}$, which is isomorphic to $k^{d}$ as a $k$-vector space. The matrix of $\rho(\eta)$ is the trans- 
pose of the matrix corresponding to $\tau(\eta)$. It follows easily from the HochschildSerre spectral sequence for group extensions that

$$
H^{*}\left(H \ltimes_{\tau} E_{d}\right) \simeq\left(k\left[y_{1}, \ldots, y_{d}\right]\right)^{H},
$$

the ring of invariants under the action of $H$ [Car81]. In the next section we discuss Carlsson's method for working with this invariant subalgebra, which is difficult to study directly.

\section{REPRESENTATION THEORY}

Let $h$ be an odd number and let $d$ be the order of 2 in $\mathbf{Z} /(h)$. Then the field extensions $k(\zeta)$ and $k(\omega)$ of $k$ obtained by adjoining a primitive $h$ th root of unity and a primitive $\left(2^{d}-1\right)$ th root of unity respectively are isomorphic to each other and to the field of $2^{d}$ elements. We denote this field by $\hat{k}$. Note that $\omega$ is a generator of the multiplicative group of units $\hat{k}^{\times}$. The Galois group $\Gamma$ of the extension $\hat{k} / k$ is a cyclic group of order $d$, generated by the Frobenius map $z \mapsto z^{\gamma}=z^{2}$.

Now let $H=\langle\eta\rangle$ be a cyclic group of order $h$ acting irreducibly, as in the previous section, on $k\left[y_{1}, \ldots, y_{d}\right]$. This action extends by $\hat{k}$-linearity to $\hat{k} \otimes k\left[y_{1}, \ldots, y_{d}\right] \simeq \hat{k}\left[y_{1}, \ldots, y_{d}\right]$, and the dimension- $d$ representation on $\hat{k}\left[y_{1}, \ldots, y_{d}\right]_{(1)} \simeq \hat{k}^{d}$ is diagonalizable, as the larger field $\hat{k}$ contains the $h$ th roots of unity. More precisely, one can show that there is an additive basis $\left\{x_{0}, \ldots, x_{d-1}\right\}$ for $\hat{k}\left[y_{1}, \ldots, y_{d}\right]_{(1)}$-hence a multiplicative basis for $\hat{k}\left[y_{1}, \ldots, y_{d}\right]$-consisting of eigenvectors of the $H$-action, such that

$$
\eta\left(x_{i}\right)=\zeta^{2^{i}} x_{i} \text { for } 0 \leq i \leq d-1
$$

for some appropriate primitive $h$ th root $\zeta$ of unity (see e.g. [CS]). Henceforth we regard $\zeta$ as fixed, and choose the primitive $\left(2^{d}-1\right)$ th root $\omega$ so that $\zeta=\omega^{\left(2^{d}-1\right) / h}$. All monomials in the $\left\{x_{0}, \ldots, x_{d-1}\right\}$ are eigenvectors of $H$. It is convenient to extend the action of the Galois group $\Gamma$ of $\hat{k} / k$ to all of $\hat{k}\left[x_{0}, \ldots, x_{d-1}\right]=\hat{k}\left[y_{1}, \ldots, y_{d}\right]$ via

$$
c^{\gamma}=c^{2}, \quad c \in \hat{k} ; \quad x_{i}^{\gamma}=x_{i+1}, \quad 0 \leq i \leq d-1,
$$

where the subscripts are read, here and throughout, as integers modulo $d$. One can check that the inclusion

$$
\imath: k\left[y_{1}, \ldots, y_{d}\right] \rightarrow \hat{k}\left[x_{0}, \ldots, x_{d-1}\right]
$$

maps $k\left[y_{1}, \ldots, y_{d}\right]$ isomorphically onto $\left(\hat{k}\left[x_{0}, \ldots, x_{d-1}\right]\right)^{\Gamma}$, the invariant subalgebra. In particular, the $2^{d}-1$ linear elements of $\left(\hat{k}\left[x_{0}, \ldots, x_{d-1}\right]\right)^{\Gamma}$ are determined by the coefficient of $x_{0}$; they are given by

$$
l_{i} \stackrel{\text { def }}{=} \omega^{i} x_{0}+\omega^{2 i} x_{1}+\cdots+\omega^{2^{d-1} i} x_{d-1}=\sum_{j=0}^{d-1} \omega^{2^{j} i} x_{j}, \quad 0 \leq i \leq d-1 .
$$

From now on, we use the following abbreviations:

$$
\begin{aligned}
\mathscr{L} & =k\left[y_{1}, \ldots, y_{d}\right]_{(1)}, \quad \widehat{\mathscr{L}}=\hat{k}\left[x_{0}, \ldots, x_{d-1}\right]_{(1)}^{H}=\imath(\mathscr{L}), \\
\operatorname{Mon}(Y) & =\operatorname{Mon}\left(k\left[y_{1}, \ldots, y_{d}\right]\right), \quad \operatorname{Mon}(X)=\operatorname{Mon}\left(\hat{k}\left[x_{0}, \ldots, x_{d-1}\right]\right),
\end{aligned}
$$


and write $\operatorname{Mon}(X)^{H}$ for the elements of $\operatorname{Mon}(X)$ which are fixed by the action of $H$ on $\hat{k}\left[x_{0}, \ldots, x_{d-1}\right]$.

\section{INVARIANT THEORY}

To discuss the $H$-invariants of $\hat{k}\left[x_{0}, \ldots, x_{d-1}\right]$, we introduce some terminology.

Notation. As in [CS], we refer to monomials of the form $x_{i}^{2^{j}}$ as "atoms." Every monomial $\mu \in \hat{k}\left[x_{0}, \ldots, x_{d-1}\right]$ can be expressed uniquely as a product of distinct atoms, which we call the atoms of $\mu$. Campbell and Selick define the weight function $w: \operatorname{Mon}(X) \rightarrow \mathbf{Z} /\left(2^{d}-1\right)$ by declaring the weight of each atom $x_{i}^{2^{j}}$ to be $w\left(x_{i}^{2^{j}}\right)=2^{i+j}$, and requiring that $w$ be multiplicative. Evidently one has $w\left(\prod_{i=0}^{d-1} x_{i}^{e_{i}}\right)=\sum_{i=0}^{d-1} 2^{i} e_{i}$. We now define the weight vector $W(\mu)$ of a monomial with atomic decomposition $\mu=\prod_{l=1}^{n(\mu)} x_{i_{l}}^{2^{j} l}$ to be the $d$-tuplet $\left(\beta_{0}(\mu), \ldots, \beta_{d-1}(\mu)\right)$, where $\beta_{t}(\mu)$ is the number of atoms of $\mu$ with weight $2^{t}$ (cf. [Woo86]). Clearly $w(\mu) \equiv \sum_{t=0}^{d-1} \beta_{t}(\mu) 2^{t}\left(\bmod 2^{d}-1\right)$. For $\phi=\sum c_{\mu} \mu$ a polynomial, $w \in \mathbf{Z} /\left(2^{d}-1\right)$, and $W=\left(\beta_{0}, \ldots, \beta_{d-1}\right) \in \mathbf{N}^{d}$, we define $\phi_{w}=\sum\left\{c_{\mu} \mu \mid w(\mu)=w\right\}$ and $\phi_{W}=\sum\left\{c_{\mu} \mu \mid W(\mu)=W\right\}$. If $\phi=\phi_{W}$, we say $\phi$ is weight-vector pure of weight-vector $W$; similarly, $\phi$ is weight-pure of weight $w$ of $\phi=\phi_{w}$.

We now describe the $H$-invariant subalgebra of $\hat{k} \otimes k\left[y_{1}, \ldots, y_{d}\right]$ in the basis $\left\{x_{0}, \ldots, x_{d-1}\right\}$.

Lemma I.5 [Car81]. Let $\mu=x_{0}^{e_{0}} \cdots x_{d-1}^{e_{d-1}}$. Then $\mu$ is an H-eigenvector with eigenvalue $\zeta^{w(\mu)}$. In particular, $\mu$ is $H$-invariant $\Leftrightarrow w(\mu)=0$. A polynomial $\phi \in \hat{k}\left[x_{0}, \ldots, x_{d-1}\right]$ is $H$-invariant $\Leftrightarrow$ each of its monomials is $H$-invariant; the invariant subalgebra $\hat{k}\left[x_{0}, \ldots, x_{d-1}\right]^{H}$ is the subalgebra generated by the invariant monomials $\operatorname{Mon}(X)^{H}$.

Let $n \in \mathbf{N}$. As usual, we write $\alpha(n)$ for the number of 1's in the dyadic representation of $n$. Let

$$
\nu \stackrel{\text { def }}{=} \min \{\alpha(n) \mid n \equiv 0(\bmod h)\}
$$

be the minimum number of 1's in the dyadic representation of any multiple of $h$. Since $2^{d}-1 \equiv 0(\bmod h)$, we have $\nu \leq d$. In fact, it is not hard to see that $\nu=\alpha(n)$ for some multiple $n$ of $h$ with $1 \leq n \leq 2^{d}-1$. From Lemma I.5 it follows that:

Corollary I.6 (cf. [Car81]). 1. Any H-invariant monomial is composed of at least $\nu$ atoms. In particular, we have $\hat{k}\left[x_{0}, \ldots, x_{d-1}\right]_{(j)}^{H}=0$ for $j<\nu$.

2. In degree $\nu$, we have $\hat{k}\left[x_{0}, \ldots, x_{d-1}\right]_{(\nu)}^{H} \neq 0$.

Proof. Let $\mu \in \operatorname{Mon}(X)^{H}$. As the integer $\sum_{t=0}^{d-1} \beta_{t}(\mu) 2^{t} \equiv w(\mu)\left(\bmod 2^{d}-1\right)$, we have that $\sum_{t=0}^{d-1} \beta_{t}(\mu) 2^{t}$ is a multiple of $h$ by Lemma I.5, and thus

$$
\nu \leq \alpha\left(\sum_{t=0}^{d-1} \beta_{t}(\mu) 2^{t}\right) \leq \sum_{t=0}^{d-1} \alpha\left(\beta_{t}(\mu) 2^{t}\right)=\sum_{t=0}^{d-1} \alpha\left(\beta_{t}(\mu)\right) \leq \sum_{t=0}^{d-1} \beta_{t}(\mu),
$$


the last term being the number of atoms of $\mu$. This proves this first statement. Now let $n, 1 \leq n \leq 2^{d}-1$, be a multiple of $h$ such that $\nu=\alpha(n)$; write $n=$ $\sum_{t=0}^{d-1} \varepsilon_{i} 2^{i}$ with $\varepsilon_{i} \in\{0,1\}$. Then the monomial $x_{0}^{\varepsilon_{0}} \cdots x_{d-1}^{\varepsilon_{d-1}}$ is $H$-invariant and has degree $\nu$.

Suppose now that $\mu \in \operatorname{Mon}(X)^{H}$ is the square of some monomial $\lambda \in$ $\operatorname{Mon}(X)$. Then $\beta_{t-1}(\lambda)=\beta_{t}(\mu)$ for all $t$, where again the subscripts are read modulo $d$, so that

$$
w(\lambda)=\sum_{t=0}^{d-1} \beta_{t}(\lambda) 2^{t}=2^{-1} \sum_{t=0}^{d-1} \beta_{t}(\mu) 2^{t}=2^{-1} w(\mu) .
$$

Since 2 is a unit in $\mathbf{Z} /\left(2^{d}-1\right)$, this means that $w(\lambda)=0$, so that $\lambda \in \operatorname{Mon}(X)^{H}$. In view of the previous lemma and equation (1), we have

Lemma $I .7$ (cf. [Car81]). Suppose $\theta \in\left(\hat{k}\left[x_{0}, \ldots, x_{d-1}\right]\right)^{H}$ satisfies $\theta=\psi^{2}$ for some $\psi \in \hat{k}\left[x_{0}, \ldots, x_{d-1}\right]$. Then $\psi \in\left(\hat{k}\left[x_{0}, \ldots, x_{d-1}\right]\right)^{H} ; \theta$ is a square in $\left(\hat{k}\left[x_{0}, \ldots, x_{d-1}\right]\right)^{H}$.

\section{SteEnRod Algebra}

Recall that the cohomology ring of any group is naturally an algebra over the mod-2 Steenrod algebra $\mathscr{A}(2)$. In particular, $k\left[y_{1}, \ldots, y_{d}\right] \simeq H^{*}\left(E_{d}\right)$ and $\left(k\left[y_{1}, \ldots, y_{d}\right]\right)^{H} \simeq H^{*}\left(H \ltimes_{\tau} E_{d}\right)$ are $\mathscr{A}(2)$-algebras. The action of $\mathscr{A}(2)$ on $k\left[y_{1}, \ldots, y_{d}\right]$ is determined by the Cartan formula and the requirements

$$
S q^{1}\left(y_{j}\right)=y_{j}^{2} ; \quad S q^{i}\left(y_{j}\right)=0 \text { for } i \geq 1 ;
$$

the action on $H^{*}\left(H \ltimes_{\tau} E_{d}\right)$ agrees with the restriction of this action to the invariant subalgebra $\left(k\left[y_{1}, \ldots, y_{d}\right]\right)^{H}$. We now recall a few pertinent facts about $\mathscr{A}(2)$ and its action on $k\left[y_{1}, \ldots, y_{d}\right]$. Let $\phi \in k\left[y_{1}, \ldots, y_{d}\right]$ and $a \in \mathbf{N}$.

Fact I.8. 1. The algebra $\mathscr{A}(2)$ is generated by the $S q^{2^{i}}$; in particular, $S q^{a}$ may be expressed in terms of $\left\{S q^{2^{i}} \mid 2^{i} \leq a\right\}$.

2. We have

$$
S q^{2^{i}}\left(\phi^{2^{j}}\right)= \begin{cases}\left(S q^{2^{i-j}} \phi\right)^{2^{j}}, & i \geq j, \\ 0, & i<j .\end{cases}
$$

3. $S q^{2^{i}}$ acts as a derivation on $\left(2^{i}\right)$ th powers.

4. $S q^{a}(\phi)=0$ for $a>\operatorname{deg}(\phi)$.

As in [Car81], we recall the definition of the Milnor primitives

$$
Q_{1}=S q^{1}, \quad Q_{i+1}=\left[S q^{2^{i}}, Q_{i}\right],
$$

and define $Q_{0}: k\left[y_{1}, \ldots, y_{d}\right] \rightarrow k\left[y_{1}, \ldots, y_{d}\right]$ by the requirements that $Q_{0}\left(y_{j}\right)$ $=y_{j}$ and that $Q_{0}$ be a derivation.

\section{Proposition I.9 [AW80].}

1. $Q_{i}=\sum_{j=1}^{d} y_{j}^{2^{i}} \partial / \partial y_{j}$ for $i \geq 0$; in particular, $Q_{i}$ is a derivation for all $i$.

2. For $i \geq d$, there exist polynomials $\phi_{i j} \in k\left[y_{1}, \ldots, y_{d}\right], 0 \leq j \leq d-1$, such that $Q_{i}=\sum_{j=0}^{d-1} \phi_{i j} Q_{j}$.

3. $Q_{0}(z)=0$ when $\operatorname{deg}(z)$ is even; $Q_{0}(z)=z$ when $\operatorname{deg}(z)$ is odd. 
Proposition I.10 [AW80]. If $\theta \in k\left[y_{1}, \ldots, y_{d}\right]$ (resp. $\left.\left(k\left[y_{1}, \ldots, y_{d}\right]\right)^{H}\right)$ satisfies $Q_{i}(\theta)=0$ for $0 \leq j \leq d-1$, then $\theta$ is the square of an element in $k\left[y_{1}, \ldots, y_{d}\right]\left(\right.$ resp. $\left.\left(k\left[y_{1}, \ldots, y_{d}\right]\right)^{H}\right)$.

Following [Car81], we extend the action of $\mathscr{A}(2)$ by $\hat{k}$-linearity to all of $\hat{k}\left[x_{0}, \ldots, x_{d-1}\right]$. The new action still satisfies the Cartan formula and the condition that $S q^{a}(f)=0$ for $a>\operatorname{deg}(f)$, but it is no longer true that $S q^{a}(f)=f^{2}$ whenever $\operatorname{deg}(f)=a$. Carlsson characterizes the new action as follows:

Proposition I.11 [Car81]. With the notation as above, the action of $\mathscr{A}(2)$ on $\hat{k}\left[x_{0}, \ldots, x_{d-1}\right]$ is determined by $\hat{k}$-linearity, the Cartan formula, and the condition that $S q^{1}\left(x_{j}\right)=x_{j-1}^{2}$.

It is straightforward to verify inductively that $Q_{j}\left(x_{i}\right)=x_{i-j}^{2^{j}}$ for $j \geq 1$. The $Q_{j}$ being derivations, we have that

$$
Q_{j}=\sum_{i=0}^{d-1} x_{i-j}^{2^{j}} \frac{\partial}{\partial x_{i}} .
$$

We summarize the statements " $Q_{j}=\sum_{i=0}^{d-1} x_{i-j}^{2^{j}} \partial / \partial x_{i}, 0 \leq j \leq d-1$ " in matrix notation as follows:

$$
\left(\begin{array}{cccc}
x_{0} & x_{1} & \ldots & x_{d-1} \\
x_{d-1}^{2} & x_{0}^{2} & \ldots & x_{d-2}^{2} \\
\vdots & \vdots & \vdots & \vdots \\
x_{1}^{2^{d-1}} & x_{2}^{2^{d-1}} & \ldots & x_{0}^{2^{d-1}}
\end{array}\right)\left(\begin{array}{c}
\frac{\partial(\theta)}{\partial x_{0}} \\
\frac{\partial(\theta)}{\partial x_{1}} \\
\vdots \\
\frac{\partial(\theta)}{\partial x_{d-1}}
\end{array}\right)=\left(\begin{array}{c}
Q_{0}(\theta) \\
Q_{1}(\theta) \\
\vdots \\
Q_{d-1}(\theta)
\end{array}\right) \text { for } \theta \in \hat{k}\left[x_{0}, \ldots, x_{d-1}\right]
$$

Note that if $\mu$ is a monomial, then $x_{i-j}^{2^{j}}\left(\partial \mu / \partial x_{i}\right)=\mu\left(x_{i-j}^{2^{j}} / x_{i}\right)$ accordingly as $x_{i}$ is or is not an atom of $\mu$. As $w\left(x_{i-j}^{2^{j}}\right)=2^{i}=w\left(x_{i}\right)$, we see that $W\left(Q_{j}(\mu)\right)=$ $W(\mu)$ provided $Q_{j}(\mu) \neq 0$. Likewise, of course, $w\left(Q_{j}(\mu)\right)=w(\mu)$.

Suppose now that $\mathscr{R}$ is a $k$-or $\hat{k}$-algebra having an $\mathscr{A}(2)$-action, e.g. $\mathscr{R}=$ $k\left[y_{1}, \ldots, y_{d}\right], \hat{k}\left[x_{0}, \ldots, x_{d-1}\right]$, or their $H$-invariant subalgebras. Recall from $\S 2$ the definition of independent sets, h.s.o.p.'s and l.s.o.p.'s of $\mathscr{R}$.

Definition I.12. Let $\mathscr{S}=\left\{\phi_{1}, \ldots, \phi_{s}\right\} \subset \mathscr{R}$ be an independent set or an h.s.o.p. $\mathscr{S}$ is $\mathscr{A}(2)$-invariant (resp. $Q$-invariant) if the ideal $\mathscr{I}\left\{\phi_{1}, \ldots, \phi_{s}\right\}$ is closed under the action of $\mathscr{A}(2)$ (resp. of the $Q_{i}, 1 \leq i<\infty$ ).

Note that $\mathscr{S}$ as above is $Q$-invariant if the ideal $\mathscr{I}\left\{\phi_{1}, \ldots, \phi_{s}\right\}$ is closed under $Q_{i}$ for $1 \leq i \leq d-1$. As $k\left[y_{1}, \ldots, y_{d}\right]$ is finitely generated over the algebra $\left(k\left[y_{1}, \ldots, y_{d}\right]\right)^{H}$, one may easily see that any $\mathscr{A}(2)$-invariant 1.s.o.p. of $\left(k\left[y_{1}, \ldots, y_{d}\right]\right)^{H}$ is also an $\mathscr{A}(2)$-invariant 1.s.o.p. of $k\left[y_{1}, \ldots, y_{d}\right]$, and maps via $l$ to an $\mathscr{A}(2)$-invariant l.s.o.p. of $\hat{k}\left[x_{0}, \ldots, x_{d-1}\right]$; similarly for $Q$ invariant l.s.o.p.'s.

\section{OUTLINE OF THE ARGUMENT}

We now outline the proof of the main theorem. In [Car81], Carlsson proves the following. 
Theorem I.13 [Car80, Car81]. Suppose $G=H \ltimes_{\tau} E_{d}$ acts freely and Z/(2)cohomologically trivially on a finite complex $X \sim_{2}\left(S^{n}\right)^{l}$. Then $H^{*}(G)=$ $\left(k\left[y_{1}, \ldots, y_{d}\right]\right)^{H}$ contains an l-element $\mathscr{A}(2)$-invariant l.s.o.p. in degree $n+1$.

This l.s.o.p. arises as follows: suppose that $G$ acts on $X$ as in Theorem I.13. Consider the Serre spectral sequence for the fibration $X \rightarrow E G \times_{G} X \rightarrow B G$, where $E G$ denotes a contractible space on which $G$ acts freely, and $B G$ the classifying space of $G$. Let $y_{j} \in H^{n}(X)$ be the generators of the exterior algebra $H^{*}(X) \simeq H^{*}\left(\left(S^{n}\right)^{l}\right)$, and let $\theta_{j} \in H^{n+1}(B G) \simeq H^{n+1}(G)$ be their transgressions in the spectral sequence. Then Carlsson shows that the set $\left\{\theta_{1}, \ldots, \theta_{l}\right\}$ forms an $\mathscr{A}(2)$-invariant 1.s.o.p. for $H^{*}(G)$ in degree $n+1$.

To prove the main theorem using Theorem I.13, our approach will be to show that the algebras $\left(k\left[y_{1}, \ldots, y_{d}\right]\right)^{H}$ possess no $\mathscr{A}(2)$-invariant l.s.o.p.'s with $d$ elements in the relevant degrees.

Notation. For $G$ as above and integers $l$ and $m$, write $P(G, l, m)$ for the following statement: Any $\mathscr{A}(2)$-invariant l.s.o.p. of $\left(k\left[y_{1}, \ldots, y_{d}\right]\right)^{H}$ of degree $m$ with $l$ elements consists entirely of squares.

Some results of [Car81] can be paraphrased to say:

Proposition 1.14. Let $m=2^{t} n$, where $n$ is odd. Suppose $P\left(G, l, 2^{i} n\right)$ is true for $1 \leq i \leq t$. Then in fact $\left(k\left[y_{1}, \ldots, y_{d}\right]\right)^{H}$ has no $\mathscr{A}(2)$-invariant l.s.o.p.' $s$ with $l$ elements in degree $m$.

Proposition $\mathrm{I} .14$ is proven by showing, first, that $\left(k\left[y_{1}, \ldots, y_{d}\right]\right)^{H}$ has no $\mathscr{A}$ (2)-invariant 1.s.o.p.'s in odd degrees, and second, that the square roots of an $\mathscr{A}(2)$-invariant l.s.o.p. consisting entirely of squares themselves form an $\mathscr{A}(2)$ invariant 1.s.o.p. In what follows, we demonstrate that if $G$ is a group of type I (resp. II), then $P(G, d, m)$ is true for all $m$ (resp. for all $m$ not of the form $\left.2^{t} \cdot 5\right)$. This together with Theorem I.13 and Proposition I.14 proves the main theorem.

The idea behind the proof that $P(G, d, m)$ holds is as follows. Let $\mathscr{S}=$ $\left\{\theta_{1}, \ldots, \theta_{d}\right\}$ be a $d$-element $\mathscr{A}(2)$-invariant l.s.o.p. for $\left(k\left[y_{1}, \ldots, y_{d}\right]\right)^{H}$ in degree $m$, with $m$ as in the main theorem. Carlsson observed that $\mathscr{A}(2)$ invariance allows us to write, for all $j=1,2, \ldots, d$ and $t=0,1, \ldots, d-1$,

$$
Q_{t}\left(\theta_{j}\right)=\sum_{k=1}^{d} q(t)_{j k} \theta_{k}
$$

for some $q(t)_{j k} \in k\left[y_{1}, \ldots, y_{d}\right]_{\left(2^{t}-1\right)}^{H}$. Let $\mathscr{Q}(t), 0 \leq t \leq d-1$, be the matrices $\left(q(t)_{j k}\right) \in \operatorname{Mat}_{d}\left(\left(k\left[y_{1}, \ldots, y_{d}\right]\right)^{H}\right)$. The goal is to prove that $\mathscr{Q}(t)=0$ for all $0 \leq t \leq d-1$; this will imply, by Proposition I.10, that each element of $\mathscr{S}$ is a square. When $G$ is of type $\mathrm{I}$, this is accomplished fairly simply by studying the elements $q(t)_{j k}$. When $G$ is of type II, the argument goes as follows: note that any $k$-basis for the $k$-linear span $\mathscr{V}\left\{\theta_{1}, \ldots, \theta_{d}\right\}$ is itself an $H$-invariant $\mathscr{A}(2)$-invariant l.s.o.p., since the ideal and subalgebra it generates are the same as those generated by $\left\{\theta_{1}, \ldots, \theta_{d}\right\}$. Thus to each basis $\mathscr{S}^{\prime}$ of $\mathscr{V}\left\{\theta_{1}, \ldots, \theta_{d}\right\}$ we may associate the matrices $\mathscr{Q}_{\mathscr{S}},(t)$ as above. Our approach is to show that $\mathscr{V}\left\{\theta_{1}, \ldots, \theta_{d}\right\}$ has a basis $\mathscr{S}^{\prime}$ for which the associated matrices are diagonal, and then, by studying the possible eigenvectors of such matrices, to verify that these diagonal matrices are in fact 0 . 
In Part II we prove some propositions concerning the action of $\mathscr{A}(2)$ on our polynomial algebras and the diagonalization of matrices, which we use in Part III to prove the main theorem. In fact, when the group $G$ is of type I, the proof that $P(G, d, m)$ holds relies only on the results of $\S 8$; the rest of the results in Part II, while interesting in their own right, are for our purposes necessary only for the discussion of groups of type II.

\section{PART II. TeChNicAl RESUltS}

\section{JOINT KERNELS OF THE $Q_{i}$. I}

Proposition I.10 says that a polynomial $\phi \in k\left[y_{1}, \ldots, y_{d}\right]$ is a square if $Q_{i}(\phi)=0$ for $0 \leq i \leq d-1$. In this section, we discuss certain polynomials $\psi$ which vanish under some, but not all, of the $Q$ 's. Namely:

Definition II.1. For $1 \leq s \leq d-1$, let

$$
\begin{aligned}
K_{s} & =\left\{\phi \in k\left[y_{1}, \ldots, y_{d}\right]_{\left(2^{s+1}-1\right)} \mid Q_{t}(\phi)=0 \text { for } 1 \leq t \leq s\right\}, \\
K_{s}^{H} & =K_{s} \cap\left(k\left[y_{1}, \ldots, y_{d}\right]\right)^{H} .
\end{aligned}
$$

In order to discuss $K_{s}$ and $K_{s}^{H}$, we introduce the following notation:

Definition II.2. For $1 \leq s \leq d-1$, let

$$
\begin{aligned}
\mathscr{T}_{s} & =\left\{\left\{y_{i_{0}}, \ldots, y_{i_{s}}\right\} \mid i_{t} \neq i_{t^{\prime}} \text { for } t \neq t^{\prime}\right\}, \\
\widehat{\mathscr{T}_{s}} & =\left\{\left\{x_{i_{0}}, \ldots, x_{i_{s}}\right\} \mid i_{t} \neq i_{t^{\prime}} \text { for } t \neq t^{\prime}\right\}, \\
\widehat{\mathscr{T}}_{s}^{H} & =\left\{\hat{\tau} \in \widehat{\mathscr{T}_{s}} \mid \sum_{j=0}^{s} 2^{i_{j}} \equiv 0(\bmod h)\right\} .
\end{aligned}
$$

Note that $\widehat{\mathscr{T}_{s}^{H}}=\varnothing$ for $1 \leq s \leq \nu-2$, where $\nu=\min \{\alpha(m) \mid m \equiv 0(\bmod h)\}$ as in $\S 5$. Moreover, if $I=\left\{i_{0}, \ldots, i_{s}\right\} \subset\left\{i_{0}, \ldots, i_{d-1}\right\}$, then the set $\tau=$ $\left\{x_{i_{j}} \mid i_{j} \in I\right\}$ belongs to $\widehat{\mathscr{T}_{s}^{H}} \Leftrightarrow \tau^{\prime}=\left\{x_{i_{k}} \mid i_{k} \notin I\right\}$ belongs to $\hat{\mathscr{T}}_{d-s-2}^{H}$, since

$$
\sum_{i_{j} \in I} 2^{i_{j}}+\sum_{i_{k} \notin I} 2^{i_{k}}=\sum_{i=0}^{d-1} 2^{i}=2^{d}-1 \equiv 0 \quad(\bmod h) .
$$

In particular, $\widehat{\mathscr{T}_{t}^{H}}=0$ for $d-\nu \leq t \leq d-2$.

Definition II.3. Let $\tau=\left(\lambda_{0}, \ldots, \lambda_{s}\right) \in \mathscr{T}_{s}$ or $\widehat{\mathscr{T}_{s}}$. Then $\mathscr{D}_{Q}(\tau)$ is the matrix

$$
D_{\mathscr{Q}}(\tau) \stackrel{\text { def }}{=}\left(\begin{array}{cccc}
\lambda_{0} & \lambda_{1} & \ldots & \lambda_{s} \\
Q_{1}\left(\lambda_{0}\right) & Q_{1}\left(\lambda_{1}\right) & \ldots & Q_{1}\left(\lambda_{s}\right) \\
\vdots & \vdots & \vdots & \vdots \\
Q_{s}\left(\lambda_{0}\right) & Q_{s}\left(\lambda_{1}\right) & \ldots & Q_{s}\left(\lambda_{s}\right)
\end{array}\right),
$$

and $\delta_{Q}(\tau)$ is the determinant $\delta\left(\mathscr{D}_{Q}(\tau)\right)$. 
Example. For $\hat{\tau}=\left\{x_{i_{0}}, \ldots, x_{i_{s}}\right\} \in \widehat{\mathscr{T}_{s}}$, we have

$$
\mathscr{D}_{Q}\left(x_{i_{0}}, \ldots, x_{i_{s}}\right)=\left(\begin{array}{cccc}
x_{i_{0}} & x_{i_{1}} & \ldots & x_{i_{s}} \\
x_{i_{0}-1}^{2} & x_{i_{1}-1}^{2} & \ldots & x_{i_{s}-1}^{2} \\
\vdots & \vdots & \vdots & \vdots \\
x_{i_{0}-s}^{2^{s}} & x_{i_{1}-s}^{2^{s}} & \ldots & x_{i_{s}-s}^{2^{s}}
\end{array}\right) .
$$

Note that the weight function is constant along each column of $\mathscr{D}_{Q}(\hat{\tau})$. Therefore the polynomial $\delta_{Q}(\hat{\tau})$ is weight-vector pure of weight-vector $W(\hat{\tau})=$ $\left(a_{0}, \ldots, a_{d-1}\right)$, where $a_{l}=1$ if $i_{j}=l$ for some $j$, and $a_{l}=0$ otherwise. Clearly $W\left(\hat{\tau}_{1}\right) \neq W\left(\hat{\tau}_{2}\right)$ if $\tau_{1} \neq \tau_{2}$. The polynomial $\delta_{Q}(\hat{\tau})$ is also of course weight-pure of weight $w=\sum_{j=0}^{s} w\left(x_{i_{j}}\right)=\sum_{j=0}^{s} 2^{i_{j}}$. In particular,

$$
\delta_{Q}(\hat{\tau}) \in\left(\hat{k}\left[x_{0}, \ldots, x_{d-1}\right]\right)^{H} \Leftrightarrow \hat{\tau} \in \widehat{\mathscr{T}}_{s}^{H} .
$$

Example. For $\tau=\left\{y_{i_{0}}, \ldots, y_{i_{s}}\right\} \in \mathscr{T}_{s}$, the definition gives

$$
\mathscr{D}_{Q}\left(y_{i_{0}}, \ldots, y_{i_{s}}\right)=\left(\begin{array}{cccc}
y_{i_{0}} & y_{i_{1}} & \ldots & y_{i_{s}} \\
y_{i_{0}}^{2} & y_{i_{1}}^{2} & \ldots & h_{i_{s}}^{2} \\
\vdots & \vdots & \vdots & \vdots \\
y_{i_{0}}^{2^{s}} & y_{i_{1}}^{2^{s}} & \ldots & y_{i_{s}}^{2^{s}}
\end{array}\right) \text {. }
$$

It is well known that the determinant of this matrix is given by the product of all the nonzero linear forms in the $y_{i_{0}}, \ldots, y_{i_{s}}$ :

$$
\delta_{Q}\left(y_{i_{0}}, \ldots, y_{i_{s}}\right)=\prod\left\{\phi \mid \phi \in k\left[y_{i_{0}}, \ldots, y_{i_{s}}\right]_{(1)}\right\} \text {. }
$$

In [Woo86], Wood gives the following description of $K_{s}$ :

Proposition II.4. $K_{s}$ is the linear span $\mathscr{V}\left\{\delta_{Q}(\tau) \mid \tau \in \mathscr{T}_{s}\right\}$.

In $\S 14$, we will need the following corollary.

Corollary II.5. Suppose $\phi \in K_{s}$. Then $Q_{s+1}(\phi)=\phi^{2}$.

Proof. As $Q_{s+1}$ and the squaring map are additive homomorphisms, it suffices to prove the corollary for $\phi=\delta_{Q}(\tau)$, where $\tau=\left(\lambda_{0}, \ldots, \lambda_{s}\right) \in \mathscr{T}_{s}$. Applying the derivation $Q_{s+1}$ one row at a time, we find that

$$
\begin{aligned}
& Q_{s+1}\left(\delta_{Q}(\tau)\right)=\left|\begin{array}{ccc}
Q_{s+1}\left(y_{i_{0}}\right) & \ldots & Q_{s+1}\left(y_{i_{s}}\right) \\
Q_{1}\left(y_{i_{0}}\right) & \ldots & Q_{1}\left(y_{i_{s}}\right) \\
\vdots & \vdots & \vdots \\
Q_{s}\left(y_{i_{0}}\right) & \ldots & Q_{s}\left(y_{i_{s}}\right)
\end{array}\right|+\left|\begin{array}{ccc}
y_{i_{0}} & \ldots & y_{i_{s}} \\
Q_{s+1}\left(Q_{1}\left(y_{i_{0}}\right)\right) & \ldots & Q_{s+1}\left(Q_{1}\left(y_{i_{s}}\right)\right) \\
\vdots & \vdots & \vdots \\
Q_{s}\left(y_{i_{0}}\right) & \ldots & Q_{s}\left(y_{i_{s}}\right)
\end{array}\right| \\
& +\cdots+\left|\begin{array}{ccc}
y_{i_{0}} & \ldots & y_{i_{s}} \\
Q_{1}\left(y_{i_{0}}\right) & \ldots & Q_{1}\left(y_{i_{s}}\right) \\
\vdots & \vdots & \vdots \\
Q_{s+1}\left(Q_{s}\left(y_{i_{0}}\right)\right) & \ldots & Q_{s+1}\left(Q_{s}\left(y_{i_{s}}\right)\right)
\end{array}\right|
\end{aligned}
$$


For $i \geq 2$, the $i$ th matrix has $i$ th row identically zero, so that its determinant vanishes. We see that

$$
Q_{s+1}\left(\delta_{Q}(\tau)\right)=\left|\begin{array}{ccc}
y_{i_{0}}^{2^{s+1}} & \ldots & y_{i_{s}}^{2^{s+1}} \\
y_{i_{0}}^{2} & \ldots & y_{i_{s}}^{2} \\
\vdots & \vdots & \vdots \\
y_{i_{0}}^{2^{s}} & \ldots & y_{i_{s}}^{2^{s}}
\end{array}\right|=\left|\begin{array}{ccc}
y_{i_{0}}^{2} & \ldots & y_{i_{s}}^{2} \\
\vdots & \vdots & \vdots \\
y_{i_{0}}^{2^{s}} & \ldots & y_{i_{s}}^{2^{s}} \\
y_{i_{0}}^{2^{s+1}} & \ldots & y_{i_{s}}^{2^{s+1}}
\end{array}\right|=\delta_{Q}(\tau)^{2},
$$

the equalities holding because in characteristic 2 , squaring is a homomorphism and the order of a matrix's rows does not affect the value of the determinant.

In order to study the invariant subspace $K_{s}^{H}$, we work in the larger field.

Definition II.6. For $1 \leq s \leq d-1$, let

$$
\begin{aligned}
\widehat{K}_{s} & =\left\{\psi \in \hat{k}\left[x_{0}, \ldots, x_{d-1}\right]_{\left(2^{s+1}-1\right)} \mid Q_{t}(\psi)=0 \text { for } 1 \leq t \leq s\right\}, \\
\widehat{K}_{s}^{H} & =\widehat{K}_{s} \cap\left(\hat{k}\left[x_{0}, \ldots, x_{d-1}\right]\right)^{H} .
\end{aligned}
$$

One can easily check, along the lines of the previous proof, that

$$
\mathscr{V}\left\{\mathscr{D}_{Q}(\hat{\tau}) \mid \hat{\tau} \in \widehat{\mathscr{S}}\right\} \subset \widehat{K}_{s} .
$$

In fact, the inclusion is an equality as we proceed to show. We need the following lemma.

Lemma II.7. Let $\tau=\left\{y_{i_{0}}, \ldots, y_{i_{s}}\right\} \in \mathscr{T}_{s}$. Then $i \delta_{Q}(\tau) \in \mathscr{V}\left\{\mathscr{D}_{Q}(\hat{\tau}) \mid \hat{\tau} \in \widehat{\mathscr{T}_{s}}\right\} \subset$ $\widehat{K}_{s}$.

Proof. Write $y_{i_{l}}=\sum_{j=0}^{d-1} \zeta^{a_{l} 2^{j}} x_{j}$ for suitable $a_{l}$ as in $\S 4$. Then

$$
{ }^{\prime} \delta_{Q}(\tau)=\left|\begin{array}{ccc}
\sum_{j=0}^{d-1} \zeta^{a_{0} 2^{j}} x_{j} & \cdots & \sum_{j=0}^{d-1} \zeta_{a_{s} 2^{j}} x_{j} \\
\left(\sum_{j=0}^{d-1} \zeta^{a_{0} 2^{j}} x_{j}\right)^{2} & \cdots & \left(\sum_{j=0}^{d-1} \zeta^{a_{s} 2^{j}} x_{j}\right)^{2} \\
\vdots & \vdots & \vdots \\
\left(\sum_{j=0}^{d-1} \zeta^{a_{0} 2^{j}} x_{j}\right)^{2^{s}} & \cdots & \left(\sum_{j=0}^{d-1} \zeta^{a_{s} 2^{j}} x_{j}\right)^{2^{s}}
\end{array}\right|
$$

I claim that each column of the above matrix is a linear combination of the column vectors $\left(x_{i}, x_{i-1}^{2}, \ldots, x_{i-s}^{2^{s}}\right)^{T}$; indeed, the $j$ th column

$$
\left(\begin{array}{c}
\sum_{i=0}^{d-1} \zeta^{a_{j} i^{i}} x_{i} \\
\left(\sum_{i=0}^{d-1} \zeta^{a_{j} 2^{i}} x_{i}\right)^{2} \\
\vdots \\
\left(\sum_{i=0}^{d-1} \zeta^{a_{j} 2^{i}} x_{i}\right)^{2^{s}}
\end{array}\right)=\sum_{i=0}^{d-1} \zeta^{a_{j} 2^{i}}\left(\begin{array}{c}
x_{i} \\
x_{i-1}^{2} \\
\vdots \\
x_{i-s}^{2^{s}}
\end{array}\right) \text {. }
$$

We can therefore express $l \delta_{Q}(\tau)$ as a linear combination of determinants of matrices with columns of the form $\left(x_{i}, x_{i-1}^{2}, \ldots, x_{i-s}^{2^{s}}\right)^{T}$, and we may of course assume that the columns of each such matrix are distinct from each other. This says exactly that $i \delta_{Q}(\tau) \in \mathscr{V}\left\{\mathscr{D}_{Q}(\hat{\tau}) \mid \hat{\tau} \in \widehat{\mathscr{S}}\right\}$.

The following corollary will be useful in the next section. Recall from $\S 4$ the notation $\mathscr{L}$ and $\widehat{\mathscr{L}}$. 
Corollary II.8. Let $\tau_{x}=\left\{x_{0}, \ldots, x_{d-1}\right\}$ and $\tau_{y}=\left\{y_{1}, \ldots, y_{d}\right\}$. Then $\delta_{Q}\left(\tau_{x}\right)$ $=i \delta_{Q}\left(\tau_{y}\right)=\prod\left\{\hat{l}_{i} \mid \hat{l}_{i} \in \widehat{\mathscr{L}}\right\}$.

Proof. As in equation (6), $\delta_{Q}\left(\tau_{y}\right)=\prod\left\{l_{i} \mid l_{i} \in \mathscr{L}\right\}$, so $i \delta_{Q}\left(\tau_{y}\right)=\prod\left\{\hat{l}_{i} \mid \hat{l}_{i} \in \widehat{\mathscr{L}}\right\}$. Since $\hat{\mathscr{T}_{d}}=\left\{\tau_{x}\right\}$, the lemma says that $0 \neq i \delta_{Q}\left(\tau_{y}\right)=c \delta_{Q}\left(\tau_{x}\right)$ for some $c \in \hat{k}$. As both $l \delta_{Q}\left(\tau_{y}\right)$ and $\delta_{Q}\left(\tau_{x}\right)$ are $\Gamma$-invariant, we see that $c=1$.

Lemma II.7 and Corollary II. 8 actually give all that is needed of the structure of $\widehat{K}_{s}$, but for the sake of completeness we prove

Proposition II.9. $\widehat{K}_{s}=\mathscr{V}\left\{\mathscr{D}_{Q}(\hat{\tau}) \mid \hat{\tau} \in \widehat{\mathscr{T}}\right\}$.

Proof. By equation (9), we must show only that $\widehat{K}_{s} \subset \mathscr{V}\left\{\mathscr{D}_{Q}(\hat{\tau}) \mid \hat{\tau} \in \widehat{\mathscr{T}_{s}}\right\}$. Let $\phi \in \widehat{K}_{s}$ and let $W_{1}, \ldots, W_{n}$ be the weight vectors corresponding to elements of $\operatorname{Mon}(\phi)$ as in $\S 5$. Since the $Q_{j}$ preserve the weight-vector classes, it is evident that $Q_{j}\left(\phi_{W_{i}}\right)=0$ for each $0 \leq j \leq s$ and $1 \leq i \leq n$. W.l.o.g., then, we may assume that $\phi$ is pure of weight vector $W$. In this case, let $\psi=\sum_{\gamma^{\prime} \in \Gamma} \phi^{\gamma^{\prime}}$. Now $\psi^{\gamma^{\prime}}=\psi$ for all $\gamma^{\prime} \in \Gamma$, so $\psi \in \imath k\left[y_{1}, \ldots, y_{d}\right]$; clearly $\psi \in \imath K_{s}$. By Lemma II.7, $\psi \in \mathscr{V}\left\{\mathscr{D}_{Q}(\hat{\tau}) \mid \hat{\tau} \in \widehat{\mathscr{T}_{s}}\right\}$; say $\psi=\sum_{i=1}^{m} c_{i} \delta_{Q}\left(\hat{\tau}_{i}\right)$. Restricting to the weight-vector class $W$, we find that

$$
\phi=\psi_{W}=\left(\sum_{i=1}^{m} c_{i} \delta_{Q}\left(\hat{\tau}_{i}\right)\right)_{W}=\sum_{i=1}^{m} c_{i}\left(\delta_{Q}\left(\hat{\tau}_{i}\right)\right)_{W} .
$$

But from the example following Definition II.3, $\left(\delta_{Q}\left(\hat{\tau}_{i}\right)\right)_{W}=\delta_{Q}\left(\hat{\tau}_{i}\right)$ if $W\left(\hat{\tau}_{i}\right)=$ $W$, and 0 else. We conclude that $W=W\left(\hat{\tau}_{i_{0}}\right)$ for some $i_{0}$, and that $\phi=$ $c_{i_{0}} \delta_{Q}\left(\hat{\tau}_{i_{0}}\right) \in \mathscr{V}\left\{\mathscr{D}_{Q}(\hat{\tau}) \mid \hat{\tau} \in \widehat{\mathscr{S}}\right\}$.

Corollary II.10. $\widehat{K}_{s}^{H}=\mathscr{V}\left\{\delta_{Q}(\hat{\tau}) \mid \hat{\tau} \in \widehat{\mathscr{T}}^{H}\right\}$.

Proof. Since the polynomials $\delta_{Q}(\hat{\tau})$ are weight-vector pure with distinct weightvectors, a linear combination of these is pure of weight $0 \Leftrightarrow$ each summand is pure of weight 0 , and hence comes from $\widehat{\mathscr{S}}^{H}$.

From Corollary II.10 and the remarks following Definition II.2, we see that $\widehat{K}_{s}^{H}$, and therefore $K_{s}^{H}$, is trivial unless $h$ has a multiple of the form $\sum_{j=0}^{s} 2^{i_{j}}$, where $0 \leq i_{j} \leq d-1$ and the $i_{j}$ are distinct. In particular:

Corollary II.11. $\widehat{K}_{s}^{H}=0$, and therefore $K_{s}^{H}=0$, for $s<\nu-1$ and $s>d-\nu-1$.

Corollary II.11 gives enough information about the action of $\mathscr{A}(2)$ on $\left(k\left[y_{1}, \ldots, y_{d}\right]\right)^{H}$ to prove that $P(G, d, m)$ holds for all $m$ when $G$ is of type I; the proof in that case may be found in $\S 15$. In the next four sections we obtain additional information about $k\left[y_{1}, \ldots, y_{d}\right]$ and this action, and about the diagonalization of matrices with entries in a polynomial ring, which will be needed in the discussion of groups of type II.

\section{JOINT KERNELS OF THE $Q_{i}$. II}

Having seen the concise description of $K_{s}$ and $\widehat{K}_{s}$ in $\S 8$, one might ask how to characterize elements of dimensions other than $2^{s+1}-1$ which lie in the joint kernel of the $Q_{i}, 1 \leq i \leq s$. It follows from Wood's proof of Proposition II.4 that: 
Lemma II.12. Suppose $\phi \in k\left[y_{1}, \ldots, y_{d}\right]_{(m)}$ (resp. $\left.\hat{k}\left[x_{0}, \ldots, x_{d-1}\right]_{(m)}\right)$ satisfies $Q_{i}(\phi)=0$ for $1 \leq i \leq s$, where $m<2^{s+1}-1$. Then $m$ is even and $\phi$ is $a$ square.

The following is a partial characterization of the joint kernel in dimensions greater than $2^{s+1}-1$ :

Let $\phi \in \hat{k}\left[x_{0}, \ldots, x_{d-1}\right]_{(m)}$, where $m \geq 2^{s+1}-1$, and suppose $\phi$ satisfies $Q_{i}(\phi)=0$ for $1 \leq i \leq s$. Let $\mu \in \operatorname{Mon}(\phi)$. Then either $\mu$ is a square or $\mu$ is of the form

$$
\mu=\left(x_{i_{0}} x_{i_{1}-1}^{2^{1}} x_{i_{2}-2}^{2^{2}} \cdots x_{i_{s}-s}^{2^{s}}\right) x_{i_{s+1}} x_{i_{s+2}} \cdots x_{i_{r}} \lambda^{2},
$$

where $\lambda \in \operatorname{Mon}(X), r \geq 0$, and the indices $i_{0}, \ldots, i_{r}$ are distinct.

Note that when $m=2^{s+1}-1$, the statement is exactly that

$$
\mu \in \operatorname{Mon}\left(\delta_{Q}\left(x_{i_{0}}, \ldots, x_{i_{s}}\right)\right),
$$

which is consistent with Proposition II.9. An analogous statement holds in the smaller polynomial algebra $k\left[y_{1}, \ldots, y_{d}\right]$, where $x_{i_{j}-j}^{2^{j}}$ is replaced by $y_{i_{j}}^{2^{j}}$.

We prove the statement for $s=1$, which is the only case needed in this paper. The other cases may be proven inductively, with a good deal of bookkeeping.

Lemma II.13. Let $\phi \in \operatorname{Ker}\left(Q_{1}\right)$, and let $\mu \in \operatorname{Mon}(\phi)$. Then either $\mu$ is a square, or $\mu$ is of the form $\mu=\left(x_{i_{0}} x_{i_{1}-1}^{2}\right) x_{i_{2}} \cdots x_{i_{r}} \lambda^{2}$ where $\lambda \in \operatorname{Mon}(X), r \geq 0$, and the indices $i_{0}, \ldots, i_{r}$ are distinct.

Proof. If $\mu$ is a square, then there is nothing to prove. If not, write $\phi=$ $\sum_{j=0}^{n} c_{\mu_{j}} \mu_{j}$, where the $\mu_{j}$ are the monomials of $\phi$; w.l.o.g. $\mu=\mu_{0}$. As $Q_{1}(\phi)=$ 0 , we must have $c_{0} Q_{1}\left(\mu_{0}\right)=\sum_{j=1}^{n} Q_{1}\left(\mu_{j}\right)$. Recall from $\S 6$ that

$$
Q_{1}\left(\mu_{0}\right)=\sum_{j=0}^{d-1} x_{j-1}^{2} \frac{\partial\left(\mu_{0}\right)}{\partial x_{j}}=\sum_{J}\left\{\frac{x_{j-1}^{2}}{x_{j}} \mu_{0}\right\},
$$

where $J=\left\{j \in \mathbf{Z} /(d) \mid \operatorname{deg}_{j}\left(\mu_{0}\right) \equiv 1(\bmod 2)\right\}$. Now $J \neq \varnothing$, as $\mu_{0}$ is not a square. Let $j_{0} \in J$. Since the monomials $\left(x_{j-1}^{2} / x_{j}\right) \mu_{0}$ of equation (11) are obviously distinct, there can be no cancellation in the right-hand summation, so that we have $x_{j_{0}-1}^{2} \mu_{0} / x_{j_{0}} \in \operatorname{Mon}\left(Q_{1}\left(\mu_{0}\right)\right)=\operatorname{Mon}\left(\sum_{j=1}^{n} c_{\mu_{j}} Q_{1}\left(\mu_{j}\right)\right)$. This means

$$
\frac{x_{j_{0}-1}^{2}}{x_{j_{0}}} \mu_{0}=\frac{x_{j_{1}-1}^{2}}{x_{j_{1}}} \mu_{1}
$$

for some $j_{1} \neq j_{0}$ and some $\mu_{1} \in \operatorname{Mon}(\phi), \mu_{1} \neq \mu_{0}$, with $\operatorname{deg}_{j_{1}}\left(\mu_{1}\right) \equiv$ $1(\bmod 2)$. Now

$$
\mu_{0}=\frac{x_{j_{1}-1}^{2}}{x_{j_{0}-1}^{2}} \frac{x_{j_{0}}}{x_{j_{1}}} \mu_{1}
$$

from which we see that $\operatorname{deg}_{j_{1}-1}\left(\mu_{0}\right) \geq 2$ and $\operatorname{deg}_{j_{1}}\left(\mu_{0}\right) \equiv \operatorname{deg}_{j_{1}}\left(\mu_{1}\right)-1 \equiv$ $0(\bmod 2)$. Since by assumption $\operatorname{deg}_{j_{0}}\left(\mu_{0}\right) \equiv 1(\bmod 2)$, we see that

$$
\chi \stackrel{\text { def }}{=} \frac{\mu_{0}}{x_{j_{1}-1}^{2} x_{j_{0}}}
$$

has $\operatorname{deg}_{j_{0}}(\chi) \equiv \operatorname{deg}_{j_{1}}(\chi) \equiv 0(\bmod 2)$. Thus $\chi$ is of the form $x_{i_{2}} \cdots x_{i_{r}} \lambda^{2}$ for some $\lambda \in \operatorname{Mon}(X)$ and some $x_{i_{2}}, \ldots, x_{i_{r}}$ distinct from each other and from $x_{j_{0}}$ and $x_{j_{1}}$. As $\mu_{0}=x_{j_{1}-1}^{2} x_{j_{0}} \chi$, this proves the lemma. 


\section{LINEAR FORMS AND $2^{t}$-POWERS}

We now digress to introduce some notation concerning linear forms and $2^{t}$ powers of polynomials, both of which play a large role in what is to follow. Let $\mathscr{L}_{1}, \ldots, \mathscr{L}_{r} \subset \mathscr{L}$ be the distinct orbits of the $H$-action on $\mathscr{L}$, and set $\pi_{j}=\prod_{\lambda_{i} \in \mathscr{L}_{j}} \lambda_{i}$, for $1 \leq j \leq r$.

Definition II.14. A polynomial $\pi \in k\left[y_{1}, \ldots, y_{d}\right]$ is a product over orbits of $H$ on $\mathscr{L}$ if $\pi=\prod_{j=1}^{r} \pi_{j}^{a_{j}}$ for suitable $a_{j}$. If $l$ of the integers $a_{j}$ are odd, then $\pi$ is an l-orbit nonsquare.

Putting hats on everything in sight, we make a similar definition for polynomials of $\hat{k}\left[x_{0}, \ldots, x_{d}\right]$.

Clearly any nontrivial $H$-invariant product of linear factors in $k\left[y_{1}, \ldots, y_{d}\right]$ (resp. $\left.\left(\hat{k}\left[x_{0}, \ldots, x_{d-1}\right]\right)^{H}\right)$ is a product over orbits of $H$ on $\mathscr{L}$ (resp. $\left.\widehat{\mathscr{L}}\right)$; the degrees of such products are multiples of $h$. From now on, we reserve the letters $\pi$ and $\rho$ for products of linear factors.

In what follows, $\mathscr{C}$ stands for either $k\left[y_{1}, \ldots, y_{d}\right]$ or $\hat{k}\left[x_{0}, \ldots, x_{d-1}\right]$, and $t$ is a positive integer. We say a polynomial $\psi \in \mathscr{C}$ is a $\left(2^{t}\right)$ th power if $\psi=\xi^{2^{t}}$ for some $\xi \in \mathscr{C}$. Any polynomial $\phi \in \mathscr{C}$ can be written, uniquely up to scalars, as $\phi=\psi \chi$, where $\psi$ is a $\left(2^{t}\right)$ th power and $\chi$ has no factors of multiplicity $2^{t}$. Furthermore, there exists up to scalars a unique $\left(2^{t}\right)$ th power polynomial $\xi \in \mathscr{C}$ of least degree such that $\phi \mid \xi$. We make the following definition:

Definition II.15. Let $t, \phi, \psi, \chi$, and $\xi$ be as above. We write $\phi^{[t]}$ for $\chi$ and $\phi^{\{t\}}$ for $\xi$, and define $\phi^{\langle t\rangle}$ by $\phi^{\langle t\rangle} \cdot \phi=\phi^{\{t\}}$.

To illustrate this notation, we list a few facts which will be useful later.

Fact II.16. Let $\phi \in \mathscr{C}$, and suppose that $\beta \in \mathscr{C}$ is a $\left(2^{t}\right)$ th power polynomial such that $\phi \mid \beta$. Then $\phi^{\{t\}} \mid \beta$, so that $\beta=\phi^{\{t\}} \alpha^{2^{t}}$ for some $\alpha \in \mathscr{C}$.

Lemma II.17. Suppose $t$ is a positive integer and $1 \neq \pi \in \mathscr{C}^{H}$ is an $H$-invariant product of linear factors, not a $\left(2^{t}\right)$ th power, such that $\operatorname{deg}(\pi) \equiv 0\left(\bmod 2^{t}\right)$. Then $\operatorname{deg}(\pi) \geq 2^{t} h$ and $\operatorname{deg}\left(\pi^{\langle t\rangle}\right) \geq 2^{t} h$.

Proof. In fact $0 \neq \operatorname{deg}(\pi) \equiv 0\left(\bmod 2^{t} h\right)$, by the remark following Definition II.14. Since $\phi^{\{t\}}$ is a $\left(2^{t}\right)$ th power and an $H$-invariant product of linear factors, we have $\operatorname{deg}\left(\phi^{\{t\}}\right) \equiv 0\left(\bmod 2^{t} h\right)$, so that $0 \neq \operatorname{deg}\left(\phi^{\langle t\rangle}\right)=\operatorname{deg}\left(\phi^{\{t\}}\right)-\operatorname{deg}(\phi) \equiv$ $0\left(\bmod 2^{t} h\right)$.

\section{EigenVeCtors OF STEENROD OPERATIONS}

In this section, $\mathscr{C}$ stands for $k\left[y_{1}, \ldots, y_{d}\right], \hat{k}\left[x_{0}, \ldots, x_{d-1}\right]$, or their $H$ invariant subalgebras.

Definition II.18. Let $\Psi \in \mathscr{A}(2)$ be a Steenrod operation of degree $j$, and let $\theta \in \mathscr{C}$ be a polynomial. If $\theta$ divides $\Psi(\theta)$ in the polynomial algebra $\mathscr{C}$, we say that $\theta$ is a $\Psi$-eigenvector with eigenvalue $\lambda_{\Psi, \theta}=\Psi(\theta) / \theta \in \mathscr{C}_{(j)}$.

Definition II.19. If $\theta \in \mathscr{C}$ is a $S q^{2^{i}}$-eigenvector for all $0 \leq i<\infty$ (resp. a $Q_{i}$-eigenvector for $0 \leq i<\infty$ ), we say $\theta$ is an $\mathscr{A}(2)$-eigenvector (resp. a $Q$-eigenvector). 
From Proposition I.9 it is evident that $\theta$ is a $Q$-eigenvector if it is a $Q_{i^{-}}$ eigenvector for $0 \leq i \leq d-1$; from Fact I.8, $\theta$ is an $\mathscr{A}(2)$-eigenvector if it is a $S q^{2^{i}}$-eigenvector for all $i$ such that $2^{i} \leq \operatorname{deg}(\theta)$. Proposition $\mathrm{I} .10$ may be restated as follows: Let $\theta \in k\left[y_{1}, \ldots, y_{d}\right]$ be a $Q$-eigenvector with $\lambda_{Q_{i}, \theta}=0$ for $0 \leq i \leq d-1$. Then $\theta$ is a square.

The following is a corollary to a theorem of Serre:

Proposition II.20 [Ser65b]. $\theta \in k\left[y_{1}, \ldots, y_{d}\right]$ is an $A(2)$-eigenvector $\Leftrightarrow \theta=$ $\prod_{l_{i} \in \mathscr{L}} l_{i}^{a_{i}}$ is a product of linear forms.

Products of $\Gamma$-invariant linear forms of $\hat{k}\left[x_{0}, \ldots, x_{d-1}\right]$ are therefore eigenvectors for the action of $\mathscr{A}(2)$ on $\hat{k}\left[x_{0}, \ldots, x_{d-1}\right]$.

The next two propositions describe, respectively, $Q$-eigenvectors of the algebra $\hat{k}\left[x_{0}, \ldots, x_{d-1}\right]$ whose eigenvalues are not necessarily 0 , and polynomials of $\hat{k}\left[x_{0}, \ldots, x_{d-1}\right]$ which are $S q^{i^{i}}$-eigenvectors for $i \leq$ some integer $c$.

Proposition II.21. 1. $\theta \in \hat{k}\left[x_{0}, \ldots, x_{d-1}\right]$ is a Q-eigenvector $\Leftrightarrow \theta=\psi^{2} \pi$, where $\psi \in \hat{k}\left[x_{0}, \ldots, x_{d-1}\right]$ is a polynomial and $\pi$ is a square-free product of $\Gamma$-invariant linear forms.

2. $\theta \in\left(\hat{k}\left[x_{0}, \ldots, x_{d-1}\right]\right)^{H}$ is a Q-eigenvector $\Leftrightarrow \theta=\psi^{2} \pi$, where $\psi \in$ $\left(\hat{k}\left[x_{0}, \ldots, x_{d-1}\right]\right)^{H}$ and $\pi$ is a product over orbits of $H$ on $\widehat{\mathscr{L}}$.

Proof. One direction of these implications is an immediate consequence of the remark following Proposition II.20 and the fact that the $Q_{i}$ 's are derivations. Suppose now that $\theta \in \hat{k}\left[x_{0}, \ldots, x_{d-1}\right]$ is a $Q$-eigenvector with $\operatorname{deg}(\theta)>1$. Then by equation (3) we have

$$
\left(\begin{array}{cccc}
x_{0} & x_{1} & \ldots & x_{d-1} \\
x_{d-1}^{2} & x_{0}^{2} & \ldots & x_{d-2}^{2} \\
\vdots & \vdots & \vdots & \vdots \\
x_{1}^{2^{d-1}} & x_{2}^{2^{d-1}} & \ldots & x_{0}^{2^{d-1}}
\end{array}\right)\left(\begin{array}{c}
\frac{\partial(\theta)}{\partial x_{0}} \\
\frac{\partial(\theta)}{\partial x_{1}} \\
\vdots \\
\frac{\partial(\theta)}{\partial x_{d-1}}
\end{array}\right)=\left(\begin{array}{c}
Q_{0}(\theta) \\
Q_{1}(\theta) \\
\vdots \\
Q_{d-1}(\theta)
\end{array}\right)=\left(\begin{array}{c}
\lambda_{0} \theta \\
\lambda_{1} \theta \\
\vdots \\
\lambda_{d-1} \theta
\end{array}\right)
$$

for appropriate $\lambda_{i} \in \hat{k}\left[x_{0}, \ldots, x_{d-1}\right]_{\left(2^{i}-1\right)}$. In the notation of $\S 14$, the matrix on the left is $\mathscr{D}_{Q}\left(\tau_{x}\right)$. By Corollary II.8, its determinant is $\delta_{Q}\left(\tau_{x}\right)=\prod_{\hat{l}_{i} \in \hat{\mathscr{L}} \hat{l}_{i}}$, which is invertible in the field of fractions $\hat{k}\left(x_{0}, \ldots, x_{d-1}\right)$ of $\hat{k}\left[x_{0}, \ldots, x_{d-1}\right]$. We now use Kramer's Rule to solve for $\left\{\partial(\theta) / \partial x_{j}\right\}_{j=0}^{d-1}$ in terms of $\left\{\lambda_{i} \theta\right\}_{i=0}^{d-1}$. Let $\mathscr{X}_{j}$ be the matrix obtained by replacing the $j$ th column of $\mathscr{D}_{Q}\left(\tau_{x}\right)$ with the vector $\left(\lambda_{0} \theta, \lambda_{1} \theta, \ldots, \lambda_{d-1} \theta\right)^{T}$; by Kramer's Rule, we have

$$
\frac{\partial(\theta)}{\partial x_{j}}=\frac{\delta\left(\mathscr{X}_{j}\right)}{\delta_{Q}\left(\tau_{x}\right)}
$$

The determinant $\delta\left(\mathscr{X}_{j}\right)$ is a homogeneous polynomial of degree $2^{d}-1$ $+\operatorname{deg}(\theta)-1=2^{d}+\operatorname{deg}(\theta)-2$. Since each coefficient of the $j$ th column of $\mathscr{X}_{j}$ is a multiple of $\theta$, we find by expanding $\delta\left(\mathscr{X}_{j}\right)$ along the $j$ th column that $\delta\left(\mathscr{X}_{j}\right)$ is itself a multiple of $\theta: \delta\left(\mathscr{X}_{j}\right)=\theta \delta_{j}$ for some polynomial $\delta_{j}$ of degree $2^{d}-2$. Thus we have

for all $1 \leq j \leq d$.

$$
\frac{\partial(\theta)}{\partial x_{j}}=\frac{\theta \delta_{j}}{\delta_{Q}\left(\tau_{x}\right)}
$$


If $\partial(\theta) / \partial x_{j}=0$ for all $j$, then $\theta$ is a square by equation (1) of $\S 2$, and part 1 of the proposition is true. Suppose then that $\partial(\theta) / \partial x_{j} \neq 0$ for some $j$. Since $\partial(\theta) / \partial x_{j}$ belongs to the ring $\hat{k}\left[x_{0}, \ldots, x_{d-1}\right]$, each factor $\hat{l}_{i}$ of the denominator $\delta_{Q}\left(\tau_{x}\right)$ must divide the numerator $\theta \delta_{j}$. For degree reasons $\delta_{Q}\left(\tau_{x}\right)$ cannot divide $\delta_{j}$, so we see that $\delta_{Q}\left(\tau_{x}\right)$ and $\theta$ must have a common factor. Therefore $\theta$ has a $\Gamma$-invariant linear factor; say $\theta=\hat{l} \theta^{\prime}$ for $\hat{l} \in \widehat{\mathscr{L}}$, $\theta^{\prime} \in \hat{k}\left[x_{0}, \ldots, x_{d-1}\right], \operatorname{deg}\left(\theta^{\prime}\right)=\operatorname{deg}(\theta)-1$. I claim now that $\theta^{\prime}$ is also a $Q$-eigenvector. Indeed, for $0 \leq i \leq d-1$, we have $0 \equiv Q_{i}(\theta)=Q_{i}(\hat{l}) \theta^{\prime}+$ $\hat{l} Q_{i}\left(\theta^{\prime}\right)\left(\bmod \hat{l} \theta^{\prime}\right)$, as $\theta$ is a $Q$-eigenvector. But $\hat{l}$, being linear and $\Gamma$-invariant, is itself a $Q$-eigenvector, so that $Q_{i}(\hat{l}) \theta^{\prime} \equiv 0\left(\bmod \hat{l} \theta^{\prime}\right)$. Consequently, for $0 \leq i \leq d-1$ we have that $\hat{l} \theta^{\prime} \mid \hat{l} Q_{i}\left(\theta^{\prime}\right)$, and so $\theta^{\prime} \mid Q_{i}\left(\theta^{\prime}\right)$. Part 1 of the proposition now follows easily by induction on $\operatorname{deg}(\theta)$.

If $\theta \in\left(\hat{k}\left[x_{0}, \ldots, x_{d-1}\right]\right)^{H}$, then one sees that the set of linear factors $\left\{\hat{l}_{i_{j}}\right\}$ of $\pi$ is closed under the action of $H$, so that $\left\{\hat{l}_{i_{j}}\right\}$ is a union of $H$-orbits and $\pi$ is $H$-invariant. It follows that $\psi$ is also $H$-invariant. This proves part 2.

In this paper, we will be concerned with $Q$-eigenvectors that lie in the subalgebra $k\left[y_{1}, \ldots, y_{d}\right] \subset \hat{k}\left[x_{0}, \ldots, x_{d-1}\right]$. The following description of such $Q$-eigenvectors follows immediately from the proposition; alternatively, it may be proven by exactly the same argument, with $\mathscr{D}_{Q}\left(\tau_{y}\right)$ and $\delta_{Q}\left(\tau_{y}\right)$ replacing $\mathscr{D}_{Q}\left(\tau_{x}\right)$ and $\delta_{Q}\left(\tau_{x}\right)$.

Proposition II.22. 1. $\theta \in k\left[y_{1}, \ldots, y_{d}\right]$ is a Q-eigenvector $\Leftrightarrow \theta=\psi^{2} \pi$, where $\psi \in k\left[y_{1}, \ldots, y_{d}\right]$ is a polynomial and $\pi$ is a square-free product of linear forms.

2. $\theta \in\left(k\left[y_{1}, \ldots, y_{d}\right]\right)^{H}$ is a Q-eigenvector $\Leftrightarrow \theta=\psi^{2} \pi$, where $\psi \in$ $\left(k\left[y_{1}, \ldots, y_{d}\right]\right)^{H}$ and $\pi$ is a product over orbits of $H$ on $\mathscr{L}$.

Proposition II.22, applied repeatedly, yields the following characterization of simultaneous eigenvectors of $S q^{2^{1}}, \ldots, S q^{2^{c}}$ in $k\left[y_{1}, \ldots, y_{d}\right]$ :

Proposition II.23. Let $c \geq d-2$.

1. A polynomial $\sigma \in k\left[y_{1}, \ldots, y_{d}\right]$ is a $S q^{2^{i}}$-eigenvector for $0 \leq i \leq c \Leftrightarrow$ $\sigma=\tau^{2^{2-d+3}} \pi$, where $\tau$ is a S $q^{2^{i}}$-eigenvector for $0 \leq i \leq d-3$ and $\pi$ is a product of linear forms.

2. A polynomial $\sigma \in\left(k\left[y_{1}, \ldots, y_{d}\right]\right)^{H}$ is a $S q^{2^{i}}$-eigenvector for $0 \leq i \leq c \Leftrightarrow$ $\sigma=\tau^{2^{c-d+3}} \pi$, where $\tau$ is an $H$-invariant $S q^{2^{i}}$-eigenvector for $0 \leq i \leq d-3$ and $\pi$ is a product over orbits of $H$ on $\mathscr{L}$.

Proof. We prove only part 1; part 2 follows exactly as did part 2 of Proposition II.21. To begin the inductive proof, suppose that $c=d-2$. Then from the definition of the $Q_{j}$ as commutators of the $S q^{2^{i}}$, we have that $\sigma$ is a $Q$-eigenvector. By Proposition II.21, $\sigma=\chi^{2} \rho$ for some polynomial $\chi$ and product $\rho$ of linear forms. We now show inductively that $\chi \mid S q^{2^{i}}(\chi)$ for $0 \leq$ $i \leq d-3$. Using the Cartan relations and Fact I.8, we write

$$
S q^{2^{i+1}}\left(\chi^{2} \rho\right)=\sum_{j=0}^{2^{i+1}} S q^{j}\left(\chi^{2}\right) S q^{2^{i+1}-j}(\rho)=\sum_{j=0}^{2^{i}}\left(S q^{j} \chi\right)^{2} S q^{2^{i+1}-2 j}(\rho) .
$$


By hypothesis, $\sigma=\chi^{2} \rho \mid S q^{2^{i+1}}\left(\chi^{2} \rho\right)$ for $0 \leq i \leq d-3$. When $i=0$, we find that

$$
\chi^{2} \rho \mid S q^{2}\left(\chi^{2} \rho\right)=\chi^{2} S q^{1}(\rho)+\left(S q^{1} \chi\right)^{2} \rho
$$

from which it follows that $\chi^{2} \mid\left(S q^{1} \chi\right)^{2}$, so that $\chi \mid S q^{1}(\chi)$.

Now assume that $\chi \mid S q^{2^{l}}(\chi)$ for $l<i \leq d-3$. By Fact I.8, we have $\chi \mid S q^{j}(\chi)$ for $j \leq 2^{i}-1$. Since $\rho$ is an eigenvector for $\mathscr{A}(2)$ by Proposition II.20, we have

$$
\chi^{2} \rho \mid\left(S q^{j} \chi\right)^{2} S q^{2^{i+1}-2 j}(\rho)
$$

for $j \leq 2^{i}-1$. From equation (12) and the hypothesis on $\sigma$, we find that

$$
0 \equiv S q^{2^{i+1}}\left(\chi^{2} \rho\right) \equiv\left(S q^{2^{i}}(\chi)\right)^{2} \rho \quad\left(\bmod \chi^{2} \rho\right)
$$

and hence that $\chi \mid S q^{2^{i}}(\chi)$. This completes the inductive step of the proof that $\chi \mid S q^{2^{i}}(\chi)$ for $0 \leq i \leq d-3$, and proves the proposition for $c=d-2$.

To prove the proposition for $c_{0}>d-2$, we assume that it holds for $d-2 \leq$ $c \leq c_{0}-1$. Suppose then that $\sigma \mid S q^{2^{i}}(\sigma)$ for $0 \leq i \leq c_{0}$. By the proposition for $c=d-2$, we have $\sigma=\chi^{2} \rho$ for some polynomial $\chi$ and product $\rho$ of linear forms. The above argument with $c_{0}-1$ replacing $d-3$ shows that $\chi$ is a $S q^{2^{i}}$-eigenvector for $0 \leq i \leq c_{0}-1$. By the inductive hypothesis, $\chi=\tau^{2^{c_{0}-d}} \psi$, where $\tau$ is a $S q^{2^{i}}$-eigenvector for $0 \leq i \leq d-3$ and $\psi$ is a product of linear forms. This means that our original polynomial $\sigma=\tau^{2^{c-d+1}} \psi^{2} \rho$. As $\psi^{2} \rho$ is the product of linear forms, we may take $\pi=\psi^{2} \rho$, proving Proposition II.23.

\section{INDEPENDENT SETS OF EIGENVECTORS}

We now prove a proposition about independent sets consisting of simultaneous eigenvectors for the first few Steenrod squares.

Proposition II.24. Let $l$ be an integer $\geq 1$, and suppose that $\left\{\phi_{1}, \ldots, \phi_{s}\right\} \subset$ $k\left[y_{1}, \ldots, y_{d}\right]$ is an independent set such that each $\phi_{i}=\psi_{i}^{2^{l}} \pi_{i}$ for some polynomial $\psi_{i}$ and product $\pi_{i}$ of linear forms. Suppose moreover that

$$
\sum_{i=1}^{s} \beta_{i} \phi_{i}=\omega^{2^{l}}
$$

for some homogeneous polynomials $\beta_{1}, \ldots, \beta_{s}$ and $\omega$. If

$$
\operatorname{deg}\left(\beta_{i_{1}}\right)+2^{d+l-2}-2^{l-1}<\operatorname{deg}\left(\phi_{i_{2}}\right) \quad \text { for all } 1 \leq i_{1}, i_{2} \leq m,
$$

then $\beta_{i} \phi_{i}$ is a $2^{l}$-power for $1 \leq i \leq s$.

Proof. We prove the proposition one $l$ at a time. Suppose to begin with that $l=1$. As in $\S 11$, the $\phi_{i}$ 's are all $Q$-eigenvectors, so for $1 \leq i \leq s$ and $0 \leq j \leq$ $d-1$ we can define elements $\lambda_{j, i} \in k\left[y_{1}, \ldots, y_{d}\right]_{\left(2^{j}-1\right)}$ by $Q_{j}\left(\phi_{i}\right)=\lambda_{j, i} \phi_{i}$. 
For each $j, 1 \leq j \leq d-1$, applying $Q_{j}$ to equation (16) gives

$$
\begin{aligned}
0 & =\sum_{i=1}^{s} Q_{j}\left(\beta_{i} \phi_{i}\right) \\
& =\sum_{i=1}^{s} Q_{j}\left(\beta_{i}\right) \phi_{i}+\sum_{i=1}^{s} \beta_{i} Q_{j}\left(\phi_{i}\right) \\
& =\sum_{i=1}^{s}\left(Q_{j}\left(\beta_{i}\right)+\lambda_{j, i} \beta_{i}\right) \phi_{i} .
\end{aligned}
$$

This is a relation between the $\phi_{i}$, the degrees of whose coefficients satisfy

$$
\operatorname{deg}\left(Q_{j}\left(\beta_{i_{1}}\right)+\lambda_{j, i_{1}} \beta_{i_{1}}\right) \leq 2^{d-1}-1+\operatorname{deg}\left(\beta_{i_{1}}\right)<\operatorname{deg}\left(\phi_{i_{2}}\right),
$$

for all $1 \leq i_{1}, i_{2} \leq s$, by assumption. As the $\phi_{1}, \ldots, \phi_{s}$ form an independent set, this relation must be trivial by Fact I.2; that is,

$$
0=\left(Q_{j}\left(\beta_{i}\right)+\lambda_{i, j} \beta_{i}\right) \phi_{i}=Q_{j}\left(\beta_{i} \phi_{i}\right), \quad 1 \leq i \leq m, 0 \leq j \leq d-1 .
$$

By Proposition I.10, we conclude that $\beta_{i} \phi_{i}$ is a square for all $i$. This proves the proposition in the case $l=1$.

To prove the proposition for general $l$, suppose inductively that it holds for all numbers $\leq l-1$, and that $\beta_{i}, \phi_{i}$, and $\omega$ satisfy the hypotheses of the proposition for the case of $l$. Then the proposition for the case of 1 says that $\beta_{i} \phi_{i}$ is a square for $1 \leq i \leq s$, so that, as in $\S 10$, there exist polynomials $\left\{\beta_{i}^{\prime}\right\}_{i=1}^{s}$ such that $\beta_{i} \phi_{i}=\left(\beta_{i}^{\prime}\right)^{2} \phi_{i}^{\{1\}}=\left(\beta_{i}^{\prime}\right)^{2} \psi_{i}^{2^{l}} \pi_{i}^{\{1\}}$. Now by construction, $\pi_{i}^{\{1\}}$ is a square; say $\pi_{i}^{\{1\}}=\left(\pi_{i}^{\prime}\right)^{2}$. The prime factors of $\left(\pi_{i}^{\prime}\right)^{2}$, being the factors of $\pi_{i}^{(1)}$, are linear forms. Set $\phi_{i}^{\prime}=\psi_{i}^{2^{l-1}} \pi_{i}^{\prime}$; then $\left(\phi_{i}^{\prime}\right)^{2}=\phi_{i}^{\{1\}}$ and $\left(\beta_{i}^{\prime} \phi_{i}^{\prime}\right)^{2}=\beta_{i} \phi_{i}$.

We now verify that the conditions of the proposition for the case of $l-1$ are satisfied with $\phi_{i}$ replaced by $\phi_{i}^{\prime}$ and $\beta_{i}$ by $\beta_{i}^{\prime}$. Square roots are unique in characteristic 2 , so from the last equality we have $\omega^{2^{2-1}}=\sum_{i=1}^{s} \beta_{i}^{\prime} \phi_{i}^{\prime}$. It remains to check the degree condition. But for each $i_{1}$ and $i_{2}$, we have $\operatorname{deg}\left(\beta_{i_{1}}^{\prime}\right) \leq$ $\frac{1}{2} \operatorname{deg}\left(\beta_{i_{1}}\right)$ and $\operatorname{deg}\left(\phi_{i_{2}}^{\prime}\right) \geq \frac{1}{2} \operatorname{deg}\left(\phi_{i_{2}}\right)$, so that the inequality

$$
\operatorname{deg}\left(\beta_{i_{1}}^{\prime}\right)+2^{d+(l-3)}-2^{l-2}<\operatorname{deg}\left(\phi_{i_{2}}^{\prime}\right), \quad 1 \leq i_{1}, i_{2} \leq m,
$$

follows from equation (17). The proposition for the case of $l-1$ then gives that $\beta_{i}^{\prime} \phi_{i}^{\prime}$ is a $2^{l-1}$-power for all $i$. But $\left(\beta_{i}^{\prime} \phi_{i}^{\prime}\right)^{2}=\beta_{i} \phi_{i}$, and consequently $\beta_{i} \phi_{i}$ is a $2^{l}$-power for $1 \leq i \leq s$. This completes the induction step and proves the proposition.

Corollary II.25. Suppose $\phi_{1}, \ldots, \phi_{s}, \omega$, and $\beta_{1}, \ldots, \beta_{s}$ are as in the proposition. Then for each $i$, either $\beta_{i}=0$ or $\operatorname{deg}\left(\beta_{i}\right) \geq \operatorname{deg}\left(\pi_{i}^{\langle l\rangle}\right)$.

Proof. By the proposition, $\beta_{i} \phi_{i}$ is a $2^{l}$-power, so $\pi_{i}^{\langle l\rangle}$ divides $\beta_{i}$ as in $\S 10$.

\section{Diagonalization}

In this section, we prove that a matrix over $k\left[y_{1}, \ldots, y_{d}\right]$ satisfying a certain simple condition is diagonalizable. The argument is due to John Conway. 
Definition II.26. Let $\mathscr{R}$ be a ring and suppose $A=\left(a_{i j}\right) \in \operatorname{Mat}_{n}(\mathscr{R})$. Then $A^{[2]}$ denotes the component-wise square of $A ; A^{[2]}=\left(a_{i j}^{2}\right)$.

Proposition II.27. Suppose $A \in \operatorname{Mat}_{n}\left(k\left[y_{1}, \ldots, y_{d}\right]\right)$ satisfies $A^{[2]}=A^{2}$. Then $A$ is diagonalizable over $k$; that is, $k^{n} \subset k\left[y_{1}, \ldots, y_{d}\right]^{n}$ has a basis consisting of eigenvectors of $A$.

Proof. Let $\mathscr{C}=\overline{k\left[y_{1}, \ldots, y_{d}\right]}$, the algebraic closure of $k\left[y_{1}, \ldots, y_{d}\right]$. Let $\sigma: \mathscr{C} \rightarrow \mathscr{C}$ be the Frobenius automorphism given by $z \mapsto z^{\sigma}=z^{2}$; this automorphism acts in the obvious way on $\operatorname{Mat}_{n}(\mathscr{C})$. Then the fixed field $\mathscr{C}^{\sigma}=k$, and the hypothesis on $A$ is that $A^{\sigma}=A^{2}$. Regarded as an element of $\operatorname{Mat}_{n}(\mathscr{C})$ acting on $\mathscr{C}^{n}, A$ can be put in Jordan-canonical form. In particular, $\mathscr{C}^{n}$ has a basis consisting of higher-order eigenvectors of $A$, that is, of vectors $v$ satisfying $(A+\lambda I d)^{\delta} v=0$ for a suitable $\lambda \in \mathscr{C}$ and integer $\delta$, where $I d$ is the identity matrix of $\operatorname{Mat}_{n}(\mathscr{C})$. I claim that any $\sigma$-invariant higher-order eigenvector $v$ of $A$ is in fact an eigenvector: note that the hypothesis on $A$ implies that $(A+\lambda I d)^{\sigma^{i}}=(A+\lambda I d)^{2^{i}}$ for all $i \geq 0$, since $\lambda^{\sigma}=\lambda^{2}$ and $A$ commutes with $I d$. Let $d$ be the smallest integer for which $(A+\lambda I d)^{2^{d}} v=0$; suppose $d \geq 1$. Then

$$
0=(A+\lambda I d)^{2^{d}} v=\left((A+\lambda I d)^{2^{d-1}}\right)^{2} v=\left((A+\lambda I d)^{2^{d-1}} v\right)^{\sigma} ;
$$

$\sigma$ being injective, we have $(A+\lambda I d)^{2^{d-1}} v=0$, contradicting the choice of $d$. So $d=0$; that is, $v$ is an eigenvector. Therefore to prove the proposition, it will suffice to construct a basis of higher-order eigenvectors all of which lie in $k^{n}=\left(\mathscr{C}^{n}\right)^{\sigma}$.

Let $\lambda \in \mathscr{C}$ be an eigenvalue of $A$ and $v \in \mathscr{C}^{n}$ a higher-order eigenvector for which $(A+\lambda I d)^{2} v=0$, where $t \geq 0$. Let $v^{\sigma^{m+1}}$ be the first $\sigma$-image of $v$ to be $\mathscr{C}$-linearly dependent on its predecessors, so that $v^{\sigma^{m+1}}=\sum_{i=0}^{m} c_{i} v^{\sigma^{i}}$ (say) and the elements $v, v^{\sigma}, \ldots, v^{\sigma^{m}}$ are linearly independent. Now $(A+\lambda I d)^{2^{t+i}} v^{\sigma^{i}}=$ $\left[(A+\lambda I d)^{2^{t}} v\right]^{\sigma^{i}}=0$; thus the vectors $v, v^{\sigma}, \ldots, v^{\sigma^{m}}$-and hence their linear combinations-are all higher-order eigenvectors belonging to $\lambda$. I claim that this linear span $\mathscr{V}\left\{v, v^{\sigma}, \ldots, v^{\sigma^{m}}\right\}$ contains a nonzero $\sigma$-invariant element: by linear independence, the equality

$$
\sum_{i=0}^{m} a_{i} v^{\sigma^{i}}=\left(\sum_{i=0}^{m} a_{i} v^{\sigma^{i}}\right)^{\sigma}=\sum_{i=0}^{m-1} a_{i}^{\sigma} v^{\sigma^{i+1}}+a_{m}^{\sigma} \sum_{i=0}^{m} c_{i} v^{\sigma^{i}}
$$

holds when the scalars $a_{0}, \ldots, a_{m}$ satisfy the equations

$$
\begin{aligned}
& a_{m}^{2} c_{0}=a_{0}, \\
& a_{i-1}^{2}+a_{m}^{2} c_{i}=a_{i}, \quad 1 \leq i \leq m .
\end{aligned}
$$

Starting with (1) and using (2) to express $a_{0}, \ldots, a_{m}$ in terms of $a_{m}$ and $c_{0}, \ldots, c_{m}$, we find that $a_{i}=\sum_{j=0}^{i}\left(a_{m}^{2} c_{j}\right)^{2^{i-j}}$ and in particular,

$$
a_{m}=\sum_{j=0}^{m}\left(a_{m}^{2} c_{j}\right)^{2^{m-j}} \text {. }
$$

Conversely, any root of the polynomial

$$
f(x)=\sum_{j=0}^{m} c_{j}^{2^{m-j}} x^{2^{m-j+1}}+x
$$


gives rise to solutions $a_{0}, \ldots, a_{m}$ to (1) and (2). Since by their definition the $c_{0}, \ldots, c_{m}$ are not all $0, f(x)$ has a nontrivial root $a_{m}$ in algebraically-closed $\mathscr{C}$, which yields a vector $v_{1}=\sum_{i=0}^{m} a_{i} v^{\sigma^{i}}$ satisfying $v_{1}^{\sigma}=v_{1}$. By the linear independence of the $v^{\sigma^{i}}, 0 \leq i \leq m$, we have $v_{1} \neq 0$. This proves the claim.

We now proceed to construct inductively a basis of $\sigma$-invariant eigenvectors for $A$. Suppose $v_{1}, \ldots, v_{l-1}$ is a linearly independent set of such eigenvectors, with $l \leq d$. Choosing a higher-order $\lambda_{l}$-eigenvector $w$ not in $\mathscr{V}\left\{v_{1}, \ldots, v_{l-1}\right\}$, as we may since $\mathscr{C}^{n}$ is spanned by higher-order eigenvectors, we repeat the above process modulo $\mathscr{V}\left\{v_{1}, \ldots, v_{l-1}\right\}$. This gives a vector

$$
0 \neq \bar{u} \in \mathscr{C}^{n} / \mathscr{V}\left\{v_{1}, \ldots, v_{l-1}\right\}
$$

satisfying $\bar{u}^{\sigma}=\bar{u}$; say $\bar{u}=u+\mathscr{V}\left\{v_{1}, \ldots, v_{l-1}\right\}$ where $u \in \mathscr{V}\left\{w, w^{\sigma}, w^{\sigma^{2}}, \ldots\right\}$ satisfies $u^{\sigma}=u+\sum_{j=1}^{l-1} \mu_{j} v_{j}$. As we saw above, $\left(A+\lambda_{l} I d\right)^{2^{e}} u=0$ for some $e$; applying $\sigma$ to this equation, we find

$$
0=\left(A+\lambda_{l} I d\right)^{2^{e+1}}\left(u+\sum_{j=1}^{l-1} \mu_{j} v_{j}\right)=\sum_{j=1}^{l-1}\left(\lambda_{j}+\lambda_{l}\right)^{2^{e+1}} \mu_{j} v_{j},
$$

so that for each $j$, either $\mu_{j}=0$ or $\lambda_{j}=\lambda_{l}$.

If $\mu_{j}=0$ for all $j$, we have $u^{\sigma}=u$, so we may take $v_{l}=u$ and proceed with the induction. If $\mu_{j_{k}} \neq 0$ for $k=1,2, \ldots, t$, we must have $\lambda_{j_{1}}=\cdots=\lambda_{j_{t}}=$ $\lambda_{l}$. Let $\left\{\rho_{j_{k}} \mid 1 \leq k \leq t\right\}$ be solutions of $x^{2}+x=\mu_{j_{k}}$ in the algebraically-closed field $\mathscr{C}$, and set $r=\sum_{k=1}^{t} \rho_{j_{k}} v_{j_{k}}$. It is easy to check that $r$ is a higher-order $\lambda_{l}$-eigenvector and that $(u+r)^{\sigma}=u+r\left(\notin \mathscr{V}\left\{v_{1}, \ldots, v_{l-1}\right\}\right)$. We may now take $v_{l}=u+r$ and proceed with the induction.

We conclude that $A$ has a basis of eigenvectors in $\left(\mathscr{C}^{n}\right)^{\sigma}=k^{\sigma}$. This proves the proposition.

Let $G=H \ltimes_{\tau} E_{d}$ as in previous sections; recall that $H$ acts on the algebra $k\left[y_{1}, \ldots, y_{d}\right]=H^{*}\left(E_{d}\right)$ with invariant subalgebra $H^{*}(G)$. The action extends to $\operatorname{Mat}_{n}\left(k\left[y_{1}, \ldots, y_{d}\right]\right)$ by $\eta\left(a_{j k}\right)=\left(\eta a_{j k}\right)$, and $\operatorname{Mat}_{n}\left(k\left[y_{1}, \ldots, y_{d}\right]\right)^{H}=$ $\operatorname{Mat}_{n}\left(\left(k\left[y_{1}, \ldots, y_{d}\right]\right)^{H}\right)$.

Corollary II.28. Suppose that $A \in \operatorname{Mat}_{n}\left(\left(k\left[y_{1}, \ldots, y_{d}\right]\right)^{H}\right)$ satisfies $A^{2}=A^{[2]}$. Then $A$ is diagonalizable and its eigenvalues are $H$-invariant.

Proof. Let $E \in \operatorname{Mat}_{n \times n}(k)$ be the matrix taking the standard basis of $k^{n}$ to the basis $\left\{v_{j}\right\}_{j=1}^{d}$ given in the proposition, so that $A=E^{-1} \Delta E$ for $\Delta=$ $\operatorname{diag}\left\{\lambda_{1}, \ldots, \lambda_{d}\right\}$. As $H$ fixes $A$, we have

$$
E^{-1} \Delta E=A=\eta A=\left(\eta E^{-1}\right)(\eta \Delta)(\eta E)=E^{-1}(\eta \Delta) E,
$$

the last equality holding because $H$ acts trivially on

$$
\operatorname{Mat}_{n}(k) \subset \operatorname{Mat}_{n}\left(k\left[y_{1}, \ldots, y_{d}\right]\right) .
$$

So $\eta \Delta=\Delta$, proving the corollary.

\section{Part III. Proof OF The MaIN THEOREM}

\section{4. $Q$-INVARIANT INDEPENDENT SETS. I}

Let $G=H \ltimes_{\tau} E_{d}$ be a semidirect product as before. Recall from $\S 7$ the notation $P(G, l, m)$, representing the statement that any $\mathscr{A}(2)$-invariant 1.s.o.p. 
of the cohomology algebra $H^{*}(G) \simeq\left(k\left[y_{1}, \ldots, y_{d}\right]\right)^{H}$ in degree $m$ consists entirely of squares. In this section, we prove some results about $Q$ - and $\mathscr{A}(2)$ invariant independent sets which will imply that $P(G, d, m)$ holds for all $m$ when $G$ is of type I; from this the main theorem for groups $\mathscr{G}$ with subgroups of this form will follow as in $\S 7$. The rest of part 3 is devoted to proving that $P(G, d, m)$ holds for all $m$ not of the form $2^{t} \cdot 5$ when $G$ is of type II, thus completing the proof of the main theorem.

We begin by introducing some notation. Let $G=H \ltimes_{\tau} E_{d}$, and suppose that $\mathscr{S}=\left\{\theta_{1}, \ldots, \theta_{s}\right\} \subset\left(k\left[y_{1}, \ldots, y_{d}\right]\right)^{H}$ is a level $Q$-invariant independent set of degree $m$. Define $b$ by $2^{b}-1<m \leq 2^{b+1}-1$. For $1 \leq t \leq b$, we may define elements $q(t)_{j k} \in k\left[y_{1}, \ldots, y_{d}\right]_{\left(2^{t}-1\right)}^{H}$ by

$$
Q_{t}\left(\theta_{j}\right)=\sum_{j=1}^{d} q(t)_{j k} \theta_{k}
$$

as in $\S 7$; as $m>2^{b}-1$ by assumption, these elements are uniquely defined by Fact I.2. Let $\mathscr{Q}(t) \in \operatorname{Mat}_{s} k\left[y_{1}, \ldots, y_{d}\right]_{\left(2^{t}-1\right)}^{H}$ be the matrices $\mathscr{Q}(t)=\left(q(t)_{j k}\right)$, $1 \leq t \leq b$. Writing $\Theta$ to represent $\left(\theta_{1}, \ldots, \theta_{s}\right) \in\left(k\left[y_{1}, \ldots, y_{d}\right]\right)^{\times d}$, we summarize the equations in (19) with the matrix equation $\Theta \mathscr{Q}(t)=\left(\theta_{1}, \ldots, \theta_{d}\right) \mathscr{Q}(t)$ $=Q_{t}(\boldsymbol{\theta})$. In this notation, we have

$$
Q_{t} Q_{u}(\Theta)=Q_{t}(\Theta \mathscr{Q}(u))=\Theta Q_{t}(\mathscr{Q}(u))+\Theta(\mathscr{Q}(t) \mathscr{Q}(u))
$$

for $1 \leq t, u \leq b$. As the $Q_{t}$ are commuting derivations, we have $Q_{u} Q_{t}(\Theta)=$ $Q_{t} Q_{u}(\boldsymbol{\theta})$, so that

$$
\Theta Q_{u}(\mathscr{Q}(t))+\boldsymbol{\Theta}(\mathscr{Q}(u) \mathscr{Q}(t))=\Theta Q_{t}(\mathscr{Q}(u))+\boldsymbol{\Theta}(\mathscr{Q}(t) \mathscr{Q}(u)) .
$$

This is a matrix equation in $\Theta$ with coefficients of degree $2^{t}-1+2^{u}-1$. For $t, u$ such that $2^{t}+2^{u}-2<m$, Fact I.2 gives

$$
Q_{u}(\mathscr{Q}(t))+Q_{t}(\mathscr{Q}(u))=[\mathscr{Q}(t), \mathscr{Q}(u)]
$$

We obtain another equation involving $\mathscr{Q}(u)$ from the fact that $Q_{u} Q_{u}=0$ :

$$
\begin{aligned}
0 & =Q_{u} Q_{u}(\boldsymbol{\Theta})=Q_{u}(\Theta \mathscr{Q}(u)) \\
& =\boldsymbol{\Theta} \mathscr{Q}(u) \mathscr{Q}(u)+\Theta\left(Q_{u} \mathscr{Q}(u)\right) \\
& =\Theta \mathscr{Q}(u)^{2}+\Theta\left(Q_{u} \mathscr{Q}(u)\right) .
\end{aligned}
$$

If $2\left(2^{u}-1\right)<m$, then as before Fact I.2 applies to give

$$
(\mathscr{Q}(u))^{2}+Q_{u}(\mathscr{Q}(u))=0
$$

In the next two propositions, we use equations $(21)$ and (22) to prove that the elements of $Q$-invariant (resp. $\mathscr{A}(2)$-invariant) independent sets are eigenvectors for the first few $Q_{i}$ (resp. $S q^{2^{i}}$ ). Recall from $\S 5$ the definition of $\nu$.

Proposition III.1. Let $G=H \ltimes_{\tau} E_{d}$, and suppose that $\mathscr{S}=\left\{\theta_{1}, \ldots, \theta_{s}\right\}$ is a level independent set for $\left(k\left[y_{1}, \ldots, y_{d}\right]\right)^{H}$ in degree $m$. If $m>2^{\nu}-2$ and $\mathscr{S}$ is invariant under $Q_{i}$ for $0 \leq i \leq \nu-1$, then $Q_{i}(\xi)=0$ for all $\xi$ in the linear span $\mathscr{V}\left\{\theta_{1}, \ldots, \theta_{s}\right\}$ and all $i, 1 \leq i \leq \nu-1$. If in addition $m>2^{\nu+1}-2$ and $\mathscr{S}$ is invariant under $Q_{\nu}$, then $\mathscr{V}\left\{\theta_{1}, \ldots, \theta_{s}\right\}$ has a basis $\theta_{1}^{\prime}, \ldots, \theta_{s}^{\prime}$ such that for $1 \leq j \leq s, Q_{\nu}\left(\theta_{j}^{\prime}\right)=\lambda_{j} \theta_{j}^{\prime}$ for suitable $\lambda_{j} \in k\left[y_{1}, \ldots, y_{d}\right]_{\left(2^{\nu}-1\right)}^{H}$.

Proof. Suppose to begin with that $m>2^{\nu}-2$ and $\mathscr{S}$ is invariant under $Q_{i}$, $0 \leq i \leq \nu-1$. The matrices $\mathscr{Q}(t)$ discussed above are defined for $1 \leq t \leq \nu-1$. 
We have $\mathscr{Q}(1)=0$, since its coefficients lie in $k\left[y_{1}, \ldots, y_{d}\right]_{(1)}^{H}$, which is 0 by Corollary I.6. We prove inductively that $\mathscr{Q}(i)=0$ for $1 \leq i \leq \nu-1$.

Suppose that the matrices $\mathscr{Q}(t)=0$ for $t<i$, where $i \leq \nu-1$. From equation (21), we have

$$
0=Q_{t}(\mathscr{Q}(i)), \quad 1 \leq t<i .
$$

This means that $0=Q_{t}\left(q(i)_{j k}\right)$ for all $t=1, \ldots, i-1$ and $j, k=1, \ldots, s$. As $\operatorname{deg}\left(q(i)_{j k}\right)=2^{i}-1$, we have $q(i)_{j k} \in K_{i-1}^{H}$, in the notation of $\S 8$. But $i-1 \leq \nu-2$, so $K_{i-1}^{H}=0$ by Corollary II.11; therefore $\mathscr{Q}(i)=0$. This completes the inductive step. We now have that $\mathscr{Q}(i)=0$ for $1 \leq i \leq \nu-1$, so that $Q_{i}\left(\theta_{j}\right)=0$ for $0 \leq i \leq \nu-1$ and $1 \leq j \leq s$. This proves the first claim of the proposition.

Suppose now that in addition $m>2^{\nu}-2$ and $\mathscr{S}$ is invariant under $Q_{\nu}$. Applying equation (21) as before, we find that $q(\nu)_{j k} \in K_{\nu-1}^{H}$. Though we can no longer conclude that $q(\nu)_{j k}=0$, we do know from Corollary II.5 that $Q_{\nu}\left(q(\nu)_{j k}\right)=\left(q(\nu)_{j k}\right)^{2}$ for all $j, k$. This means

$$
Q_{\nu}(\mathscr{Q}(\nu))=\mathscr{Q}(\nu)^{[2]},
$$

in the notation of $\S 13$. On the other hand, from equation (22) we have

$$
Q_{\nu} \mathscr{Q}(\nu)=\mathscr{Q}(\nu)^{2}
$$

The last two equations say that $\mathscr{Q}(\nu)^{2}=\mathscr{Q}(\nu)^{[2]}$, so that $\mathscr{Q}(\nu)$ satisfies the conditions of Proposition II.27 and its corollary, as a matrix with coefficients in $k\left[y_{1}, \ldots, y_{d}\right] \supset\left(k\left[y_{1}, \ldots, y_{d}\right]\right)^{H}$. Therefore its action on $\mathscr{V}\left\{\theta_{1}, \ldots, \theta_{s}\right\}$ is diagonalizable, and the diagonalized matrix is $H$-invariant. The eigenvectors $\theta_{1}^{\prime}, \ldots, \theta_{s}^{\prime}$ forming the new basis for $\mathscr{V}\left\{\theta_{1}, \ldots, \theta_{s}\right\}$ are $H$-invariant and are killed by $Q_{1}, \ldots, Q_{\nu-1}$ as above.

Before looking at the implication of Proposition III.1 for groups of type I, we prove an analogous result describing independent sets which are invariant not under the first few $Q_{i}$ 's but under the first few $S q^{2^{i}}$ 's. Proposition III.2 will play a role later on in the discussion of groups of type II.

Proposition III.2. Let $G=H \ltimes_{\tau} E_{d}$, and suppose that $\mathscr{S}=\left\{\theta_{1}, \ldots, \theta_{s}\right\} \subset$ $\left(k\left[y_{1}, \ldots, y_{d}\right]\right)^{H}$ is a level independent set in degree $m>2^{t+1}$ where $t$ is defined by $2^{t-1}<\nu \leq 2^{t}$. Suppose $\mathscr{S}$ is invariant under $S q^{2^{i}}$ for $0 \leq i \leq$ $t$. Then $S q^{2^{i}}(\xi)=0$ for all $\xi$ in the linear span $\mathscr{V}\left\{\theta_{1}, \ldots, \theta_{s}\right\}$ and all $i$, $0 \leq i \leq t-1$. Moreover, $\mathscr{V}\left\{\theta_{1}, \ldots, \theta_{s}\right\}$ has a basis $\theta_{1}^{\prime}, \ldots, \theta_{s}^{\prime}$ such that for $1 \leq j \leq s, S q^{2^{t}}\left(\theta_{j}^{\prime}\right)=\mu_{j} \theta_{j}^{\prime}$ for suitable $\mu_{j} \in k\left[y_{1}, \ldots, y_{d}\right]_{\left(2^{t}\right)}^{H}$.

Proof. This time we define elements $p(i)_{j k} \in k\left[y_{1}, \ldots, y_{d}\right]_{\left(2^{i}\right)}^{H}$ by $S q^{2^{i}}\left(\theta_{j}\right)=$ $\sum_{k=1}^{s} p(i)_{j k} \theta_{k}$, and let $\mathscr{P}(i)$ be the matrices $\left(p(i)_{j k}\right)$; as before, these matrices are well-defined provided $0 \leq i \leq t$. By Corollary I.6, $\mathscr{P}(i)=0$ for $0 \leq i \leq$ $t-1$, so from Fact I.8 we have $S q^{a}(\Theta)=0$ for $1 \leq a \leq 2^{t}-1$. It then follows from the Adem relations that

$$
\begin{aligned}
0 & =S q^{2^{t}} S q^{2^{t}}(\boldsymbol{\Theta})=S q^{2^{t}}(\boldsymbol{\Theta} \mathscr{P}(t)) \\
& =(\boldsymbol{\Theta} \mathscr{P}(t)) \mathscr{P}(t)+\boldsymbol{\Theta}\left(S q^{2^{t}} \mathscr{P}(t)\right)=\boldsymbol{\Theta}\left(\mathscr{P}(t)^{2}+\mathscr{P}(t)^{[2]}\right) .
\end{aligned}
$$


As $2^{t+1}<m$, we conclude from this matrix equation that $0=\mathscr{P}(t)^{2}+P(t)^{[2]}$. The rest follows as in the proof of Proposition III.1.

Propositions III.1 and III.2 give information about level $Q$ - and $\mathscr{A}(2)-$ invariant independent sets of sufficiently high degree. The following special case of a result from [Car81] provides an estimate for the degrees of those level $Q$-invariant independent sets which contain $d$ elements, that is, of $Q$-invariant l.s.o.p.'s.

Proposition III.3 [Car81]. Suppose $\mathscr{S}=\left\{\theta_{1}, \ldots, \theta_{s}\right\}$ is a Q-invariant l.s.o.p. of $\left(k\left[y_{1}, \ldots, y_{d}\right]\right)^{H}$ of degree $m$. Then $2 h \mid m$.

Using this estimate and Proposition III.1, we proceed to prove the main theorem for groups $\mathscr{G}$ containing subgroups of type $\mathrm{I}$.

\section{Main Theorem, Part I}

In view of Propositions III.1 and III.3, we have the following:

Proposition III.4. Suppose $G=H \ltimes_{\tau} E_{d}$ is of type I. Then the algebra $H^{*}(G) \simeq$ $\left(k\left[y_{1}, \ldots, y_{d}\right]\right)^{H}$ has no d-element $\mathscr{A}(2)$-invariant l.s.o.p.'s.

Proof. By Proposition I.14, it suffices to prove that $P(G, d, m)$ holds for all even $m$, i.e. that any $d$-element $\mathscr{A}(2)$-invariant 1.s.o.p. of even degree $m$ consists entirely of squares. Suppose that $\mathscr{S}=\left\{\theta_{1}, \ldots, \theta_{d}\right\}$ is such an 1.s.o.p. As its degree is even, we have $Q_{0}\left(\theta_{j}\right)=0$ for $1 \leq j \leq d$. Moreover, $m>h=2^{d}-1$ by Proposition III.3, and as $G$ is of type I, $\nu=d$. It follows from Proposition III. 1 that $Q_{i}\left(\theta_{j}\right)=0$ for $1 \leq i \leq d-1$ and $1 \leq j \leq d$. By Proposition I.10, $\theta_{j}$ is a square for $1 \leq j \leq d$. This proves the proposition.

We can now prove

Theorem III.5 (Main Theorem, Part I). Suppose the group $\mathscr{G}$ contains a subgroup $G=H \ltimes_{\tau} E_{d}$ of type I. Then $\mathscr{G}$ does not act freely and $k$-cohomologically trivially on any finite complex $X \sim_{2}\left(S^{n}\right)^{d}$, for any $n$.

Proof. Immediate from Theorem I.13 and Proposition III.4.

\section{6. $Q$-INVARIANT INDEPENDENT SETS, PART II}

Let $G=H \ltimes_{\tau} E_{4}$ be a group of type II, and let $\mathscr{S}=\left\{\theta_{1}, \ldots, \theta_{s}\right\}$ be a $Q$ invariant 1.s.o.p. of $\left(k\left[y_{1}, \ldots, y_{4}\right]\right)^{H}$ in degree $m$. Proposition III.1 says that the linear span $\mathscr{V}\left\{\theta_{1}, \ldots, \theta_{s}\right\}$ has a basis $\mathscr{S}^{\prime}=\left\{\theta_{1}^{\prime}, \ldots, \theta_{s}^{\prime}\right\}$ consisting of eigenvectors for $Q_{i}, 0 \leq i \leq 2$, provided $m>6$. In the next few sections we show, first, that if $m>14$, then the $\theta_{j}^{\prime}$ are in fact eigenvectors for $Q_{3}$ as well, and are therefore $Q$-eigenvectors; and second, that if in addition $\mathscr{S}$ is actually a $\mathscr{A}(2)$-invariant l.s.o.p. and $m \neq 5 \cdot 2^{t}$ for any $t$, then these eigenvectors must in fact be squares.

Suppose then that $m>14$, and that $\mathscr{S}$ is a $Q$-invariant 1.s.o.p. in degree $m$; let $\mathscr{S}^{\prime}$ be as above. Let $\mathscr{Q}(u), 0 \leq u \leq 3$, be the matrices for the $Q_{u}$ in the basis $\left\{\theta_{1}^{\prime}, \ldots, \theta_{s}^{\prime}\right\}$ as in $\S 14$. Then $\mathscr{Q}(1)=0$ and $\mathscr{Q}(2)$ is diagonal. The degree conditions for equations (21) and (22) of $\S 14$ are now satisfied for 
$t=1,2$ and $u=3$, and these equations give

$$
\begin{aligned}
Q_{1}(\mathscr{Q}(3)) & =0 \\
Q_{2}(\mathscr{Q}(3))+Q_{3}(\mathscr{Q}(2)) & =[\mathscr{Q}(2), \mathscr{Q}(3)], \\
Q_{3}(\mathscr{Q}(3)) & =\mathscr{Q}(3)^{2} .
\end{aligned}
$$

We use these equations first to restrict the monomials that can appear in the off-diagonal elements of $\mathscr{Q}(3)$, and then to show that these elements are in fact 0 . This will mean that $\mathscr{Q}(3)$ is diagonal and the $\theta_{j}^{\prime}$ 's are $Q$-eigenvectors.

Taking the $(i, j)$ th coordinate of the matrices in equation (27) gives, for $i \neq j$,

$$
Q_{2}\left(q(3)_{i j}\right)=\left(q(2)_{i i}+q(2)_{j j}\right) q(3)_{i j}
$$

as $\mathscr{Q}(2)$ is diagonal. If $q(2)_{i i}+q(2)_{j j}=0$, then $Q_{2}\left(q(3)_{i j}\right)=Q_{1}\left(q(3)_{i j}\right)=0$, so $q(3)_{i j} \in K_{2}^{H}$, in the notation of $\S 8$. But $K_{2}^{H}=0$ by Corollary II.11, and therefore $q(3)_{i j}=0$.

We now consider the case $q(2)_{i i}+q(2)_{j j} \neq 0$, and check that $q(3)_{i j}=0$ here as well. We do so by showing that $\operatorname{Mon}\left(q(3)_{i j}\right)=\varnothing$.

Equation (26) and its analogue $Q_{1}(\mathscr{Q}(2))=0$ say that $Q_{1}\left(q(2)_{i j}\right)=Q_{1}\left(q(3)_{i j}\right)$ $=0$ for all $1 \leq i, j \leq 4$. Thus by Lemma II.13 each monomial of $q(2)_{i j}$ and $q(3)_{i j}$ is of the form $\left(x_{i_{0}} x_{i_{1}-1}^{2}\right) x_{i_{2}} \cdots x_{i_{r}} \lambda^{2}$ for suitable $x_{i_{k}}$ and $\lambda$. These monomials must all be $H$-invariant, since the polynomials $q(2)_{i j}$ and $q(3)_{i j}$ are.

Notation. For typographical convenience, we write $\left(a_{0} a_{1} \cdots a_{d-1}\right)$ to represent the monomial $x_{0}^{a_{0}} \cdots x_{d-1}^{a_{d-1}}$.

One may easily check that the only possible monomials of $q(2)_{i j}$ are $\left(\begin{array}{llll}2 & 0 & 0 & 1\end{array}\right)$ and its conjugates under $\Gamma$, and the only possibilities for $\operatorname{Mon}\left(q(3)_{i j}\right)$ are

$$
\left(\begin{array}{llll}
6 & 0 & 1 & 0
\end{array}\right), \quad\left(\begin{array}{llll}
4 & 3 & 0 & 0
\end{array}\right), \quad\left(\begin{array}{llll}
4 & 0 & 2 & 1
\end{array}\right),
$$

and their conjugates under $\Gamma$.

Notation. Henceforth, we write $p(2)_{i j}$ for $q(2)_{i i}+q(2)_{j j}$.

From the above paragraph, we have $p(2)_{i j}=\sum_{n=0}^{3} c^{\gamma^{n}}\left(\begin{array}{llll}2 & 0 & 0 & 1\end{array}\right)^{\gamma^{n}}$ for some $c \neq 0$. Equation (29) becomes

$$
Q_{2}\left(q(3)_{i j}\right)=p(2)_{i j} q(3)_{i j}
$$

We now ask which monomials may appear in equation (31). Any monomial of $Q_{2}\left(q(3)_{i j}\right)$ must be conjugate to one of

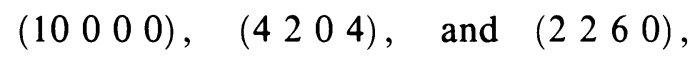

and any monomial of $p(2)_{i j} q(3)_{i j}$ is conjugate to a product of $\left(\begin{array}{llll}2 & 0 & 0 & 1\end{array}\right)$ with one of the monomials of equation (30). One may check that the monomial

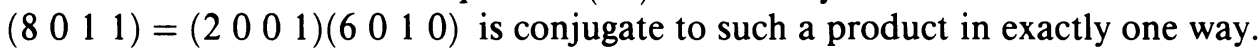
Writing $\lambda$ for the coefficient of $\left(\begin{array}{llll}6 & 0 & 1 & 0\end{array}\right)$ in $q(3)_{i j}$, we see that the coefficient

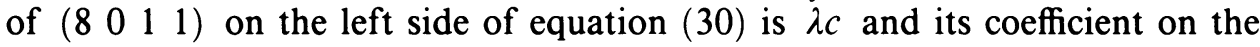
right side is 0 ; as $c \neq 0$, we conclude that $\lambda=0$. That is, $\left(\begin{array}{llll}6 & 0 & 1 & 0\end{array}\right)$, and likewise its conjugates, does not appear in $q(3)_{i j}$ after all. The same argument

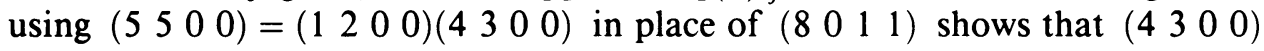
and its conjugates do not appear either, in any off-diagonal element $q(3)_{i j}$. 
It remains to eliminate the monomials $\left(\begin{array}{llll}4 & 0 & 2 & 1\end{array}\right)$ and $\left(\begin{array}{llll}3 & 2 & 2 & 0\end{array}\right)$ and their conjugates. We do so using equation (28). Looking at the $i, j$-coordinate, we find that

$$
Q_{3}\left(q(3)_{i j}\right)=\sum_{k=1}^{4} q(3)_{i k} q(3)_{k j}
$$

Recall that

$$
\begin{aligned}
& \operatorname{Mon}\left(q(3)_{a b}\right) \\
& \quad \subset\left\{\begin{array}{llll}
\left.\left\{\begin{array}{llll}
4 & 0 & 2 & 1
\end{array}\right)^{\gamma},\left(\begin{array}{llll}
3 & 2 & 2 & 0
\end{array}\right)^{\gamma} \mid \gamma \in \Gamma\right\}, \quad a \neq b, \\
\left.\left\{\begin{array}{lllll}
4 & 0 & 2 & 1
\end{array}\right)^{\gamma},\left(\begin{array}{lllll}
3 & 2 & 2 & 0
\end{array}\right)^{\gamma},\left(\begin{array}{llll}
6 & 0 & 1 & 0
\end{array}\right)^{\gamma},\left(\begin{array}{llll}
0 & 0 & 4 & 3
\end{array}\right)^{\gamma} \mid \gamma \in \Gamma\right\}, \quad a=b .
\end{array}\right.
\end{aligned}
$$

One may easily check that the monomial $\left(\begin{array}{llll}2 & 10 & 2 & 0\end{array}\right)=Q_{3}\left(\begin{array}{llll}3 & 2 & 2 & 0\end{array}\right)$ does not appear in the right-hand side of equation (32) at all. It follows that its coefficient in $Q_{3}\left(q(3)_{i j}\right)$, which is its coefficient in $q(3)_{i j}$, vanishes, and hence that $\left(\begin{array}{llll}3 & 2 & 2 & 0\end{array}\right) \notin \operatorname{Mon}\left(q\left(\begin{array}{l}3 \\ )_{i j}\end{array}\right) \subset\left\{\left(\begin{array}{llll}4 & 0 & 2 & 1\end{array}\right)^{\gamma} \mid \gamma \in \Gamma\right\}\right.$. Referring again to equation (31),

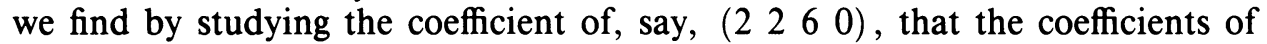

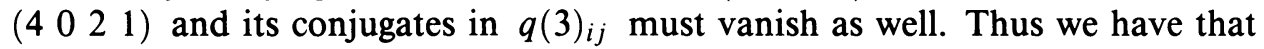
$q(3)_{i j}=0$ whenever $p(2)_{i j} \neq 0$. We have already seen that $q(3)_{i j}=0$ whenever $p(2)_{i j}=0$ and $i \neq j$, and therefore we conclude that $\mathscr{Q}(3)$ is diagonal as asserted.

Thus the $\theta_{j}^{\prime}$ are all $Q$-eigenvectors. From Corollary II.22, we have that $\theta_{j}^{\prime}=\phi_{j}^{2} \rho_{j}$ for all $j$, where $\phi_{j} \in\left(k\left[y_{1}, \ldots, y_{4}\right]\right)^{H}$ and $\rho_{j}$ is a product over orbits of $H$ on $\mathscr{L}$.

Suppose now that $m$ is even. Because $h$ is odd, each $\rho_{j}$ must be a product over an even number of orbits. Now there are only three orbits of $G$ on $\mathscr{L}$, and as the $\theta_{j}$ form an independent sequence, they have no common factors and the orbits associated to them must be distinct. Without loss of generality, then, $\theta_{j}^{\prime}=\phi_{j}^{2}$ for $j \leq s-1$, and $\theta_{s}^{\prime}=\phi_{s}^{2} \rho$ where $\rho$ is either 1 or a 2 -orbit nonsquare.

We have proven

Proposition III.6. Suppose $\left(k\left[y_{1}, \ldots, y_{4}\right]\right)^{H}$ has a $Q$-invariant independent sequence $\mathscr{S}=\left\{\theta_{1}, \ldots, \theta_{s}\right\}$ in degree $m>14$. Then there is a basis $\mathscr{S}^{\prime}=$ $\left\{\theta_{1}^{\prime}, \ldots, \theta_{s}^{\prime}\right\}$ of $\mathscr{V}\left\{\theta_{1}, \ldots, \theta_{s}\right\}$ such that $\theta_{j}^{\prime}=\phi_{j}^{2} \rho_{j}$ for $1 \leq j \leq s$, where $\rho_{j}$ is a (possibly trivial) product over orbits of $H$ on $\mathscr{L}$. If $m$ is even, then w.l.o.g. $\theta_{j}^{\prime}=\phi_{j}^{2}$ for $1 \leq j \leq s-1$, and $\theta_{s}^{\prime}=\phi_{s}^{2} \rho$ where $\rho$ is either 1 or a 2-orbit nonsquare.

The rest of Part III will be devoted to demonstrating that if $\mathscr{S}$ is a $\mathscr{A}(2)$ invariant l.s.o.p. and $m$ is not of the form $2^{t} \cdot 5$, then the second possibility cannot in fact occur. This will prove $P(G, d, m)$ for such $m$ and groups $G$ of type II.

\section{7. $\mathscr{A}(2)$-INVARIANT L.S.O.P.'s}

In this section, we prove a proposition which will later be used as the inductive step in a description of the $\mathscr{A}(2)$-invariant 1.s.o.p.'s in $H^{*}(G)$ for $G$ of type II. 
Lemma III.7. Let $G$ be a group of type II, and let $l \geq 1$ be an integer. Suppose $\mathscr{S}=\left\{\theta_{1}, \ldots, \theta_{4}\right\} \subset\left(k\left[y_{1}, \ldots, y_{d}\right]\right)^{H}$ is a $\mathscr{A}(2)$-invariant l.s.o.p. of degree $m$. Suppose that the $\theta_{j}=\psi_{j}^{2^{l}}$ are $2^{l}$-powers, $1 \leq j \leq 3$, and $\theta_{4}=\sigma^{2^{l}} \pi$ is a $2^{l}$-power times a 2-orbit nonsquare. Then for $i$ such that $0 \leq i \leq 2$ and $2^{l+i}+2^{l+2}-2^{l-1}<m$, the ideal $\mathscr{I}\left\{\theta_{1}, \theta_{2}, \theta_{3}\right\}$ is invariant under $S q^{2^{1+i}}$ (equivalently, the ideal $\mathscr{I}\left\{\psi_{1}, \psi_{2}, \psi_{3}\right\}$ is invariant under $S q^{2^{i}}$ ).

Proof. Suppose that $i$ satisfies the conditions of the lemma. Since the ideal $\mathscr{I}\left\{\theta_{1}, \ldots, \theta_{4}\right\}$ is $\mathscr{A}(2)$-invariant, there exist elements

$$
a(l+i)_{j k} \in k\left[y_{1}, \ldots, y_{4}\right]_{\left(2^{l+i}\right)}^{H}, \quad 1 \leq j \leq 3,
$$

such that

$$
\left(S q^{2^{i}} \psi_{j}\right)^{2^{l}}=S q^{2^{i+l}} \theta_{j}=\sum_{k=1}^{4} a(l+i)_{j k} \theta_{k} .
$$

We verify now that Proposition II.24 and its corollary apply to this situation. Indeed, we have

$$
\operatorname{deg}\left(a(l+i)_{j k}\right)+2^{l+(4-2)}-2^{l-1}=2^{l+i}+2^{l+2}-2^{l-1}<m,
$$

and in addition, $\operatorname{deg}\left(\pi^{\langle l\rangle}\right) \geq 2^{l} \cdot 5$ by Lemma II.17, so that $\operatorname{deg}\left(a(l+i)_{j k}\right) \leq$ $2^{l+2}<\operatorname{deg}\left(\pi^{\langle l\rangle}\right)$. Proposition II.24 and Corollary II.25 then give, for $1 \leq j \leq 3$,

$a(l+i)_{j k}=\left\{\begin{array}{l}\left(\alpha(l+i)_{j k}\right)^{2^{l}} \text { for some } \alpha(l+i)_{j k} \in k\left[y_{1}, \ldots, y_{4}\right]_{\left(2^{i}\right)}^{H}, \quad 1 \leq k \leq 3, \\ 0, \quad k=4 .\end{array}\right.$

In view of equation (33), equation (34) means that the ideal $\mathscr{I}\left(\theta_{2}, \theta_{2}, \theta_{3}\right)$ is invariant under $S q^{2^{l+i}}$ for $0 \leq i \leq 2$. Taking $\left(2^{l}\right)$ th roots of equation (33) now gives

$$
S q^{2^{i}} \psi_{j}=\sum_{k=1}^{3} \alpha(l+i)_{j k} \psi_{k}
$$

so that the ideal $\mathscr{I}\left(\psi_{1}, \psi_{2}, \psi_{3}\right)$ is invariant under $S q^{2^{i}}$.

Proposition III.8. Let $G$ be a group of type II, and let $l \geq 1$ be an integer. Suppose $\mathscr{S}=\left\{\theta_{1}, \ldots, \theta_{4}\right\} \subset\left(k\left[y_{1}, \ldots, y_{4}\right]\right)^{H}$ is a $\mathscr{A}(2)$-invariant l.s.o.p. of degree $m$ such that the $\theta_{j}=\psi_{j}^{2^{l}}$ are $2^{l}$-powers, $1 \leq j \leq 3$, and $\theta_{4}=\sigma^{2^{l}} \pi$ is a $2^{l}$-power times a 2-orbit nonsquare. If $m>14 \cdot 2^{l}$, then in fact $\theta_{j}=\phi_{j}^{2^{l+1}}$ is a $2^{l+1}$-power, $1 \leq j \leq 3$, and $\theta_{4}=\tau^{2^{l+1}} \rho$ is a $2^{l+1}$-power times a 2-orbit nonsquare.

Proof. We begin by showing that $\theta_{j}$ is a $2^{l+1}$-power, $1 \leq j \leq 3$. We may assume that $\pi$ has no factors of multiplicity $\geq 2^{l}$. Now for $0 \leq i \leq 2$ we have

$$
2^{l+i}+2^{l+2}-2^{l-1} \leq 2^{l+3}-2^{l-1}<m,
$$

so that by Lemma III.7, the ideal $\mathscr{I}\left\{\psi_{1}, \psi_{2}, \psi_{3}\right\}$ is invariant under $S q^{2^{i}}$ for $0 \leq i \leq 2$. The ideal is therefore invariant under $Q_{i}$ for $0 \leq i \leq 3$. Now the $\psi_{j}$, being $\left(2^{l}\right)$ th roots of an independent set, themselves form an independent set, the degree of which is $m / 2^{l}>14$ by hypothesis. Thus by Proposition 
III.6, there exists a basis $\left\{\psi_{1}^{\prime}, \psi_{2}^{\prime}, \psi_{3}^{\prime}\right\}$ for $\mathscr{V}\left(\psi_{1}, \psi_{2}, \psi_{3}\right)$ such that each $\psi_{j}^{\prime}$ is of the form $\psi_{j}^{\prime}=\xi_{j}^{2} \rho_{j}$ for some $\xi_{j}$ and some square-free product $\rho_{j}$ over $H$-orbits on $\mathscr{L}$. Now there are only three products of $H$-orbits on $\mathscr{L}$, and two of these divide $\theta_{4}$ by assumption. Since the $\theta_{j}$ 's form an independent set, they have no common factors, and so w.l.o.g. $\rho_{1}=\rho_{2}=1$ and $\rho_{3}$ is either 1 or a product over a single orbit, hence of odd order $h$. But since $\psi_{1}^{\prime}=\xi_{1}^{2}$ is a square, the common degree of the $\psi_{j}^{\prime}$ is even, which means that $\operatorname{deg}\left(\rho_{3}\right)$ must be even. Thus $\rho_{3}=1$ as well, so that all three of the $\psi_{j}^{\prime}$ are squares. Therefore the original $\psi_{j}$ 's, which are linear combinations of the $\psi_{j}^{\prime}$ 's, are themselves squares; say $\psi_{j}=\phi_{j}^{2}$. We conclude that for $1 \leq j \leq 3, \theta_{j}=\psi_{j}^{2^{l}}=\phi_{j}^{2^{l+1}}$ is a $2^{l+1}$-power, as asserted.

It remains to show that $\theta_{4}=\sigma^{2^{l}} \pi$ actually factors as $\theta_{4}=\tau^{2^{l+1}} \rho$ with $\rho$ a 2-orbit nonsquare. To begin with, I claim that $\sigma^{2^{l}}$ divides $S q^{2^{s}}\left(\sigma^{2^{l}}\right)$ for $0 \leq s \leq l+2$. This is proved by induction on $s$. The cases $s<l$ are trivial, as $S q^{2^{s}}$ acts trivially on $2^{s+1}$-powers by Fact I.8. Suppose now that $\sigma^{2^{l}}$ divides $S q^{2^{q}}\left(\sigma^{2^{l}}\right)$ for $q<s$. Since the Steenrod algebra $\mathscr{A}(2)$ is generated by $\left\{S q^{2^{q}}\right\}_{q=0}^{\infty}$, we have in fact that $\sigma^{2^{l}}$ divides $S q^{r}\left(\sigma^{2^{l}}\right)$ for $1 \leq r \leq 2^{s}-1$. Bearing in mind that $\pi$ is an eigenvector for $\mathscr{A}(2)$, define the polynomials $\left\{\lambda_{r}\right\}_{r=0}^{2^{s}-1}$ and $\left\{\mu_{r}\right\}_{r=0}^{s} \subset \hat{k}\left[x_{0}, \ldots, x_{d-1}\right]_{(r)}^{H}$ by

$$
S q^{r}\left(\sigma^{2^{l}}\right)=\lambda_{r} \sigma^{2^{l}} \text { and } S q^{r}(\pi)=\mu_{r} \pi .
$$

Since the ideal generated by $\mathscr{S}$ is $\mathscr{A}(2)$-invariant, we can also define elements $a(s)_{k} \in \hat{k}\left[x_{0}, \ldots, x_{d-1}\right]_{\left(2^{s}\right)}^{H}$ by $S q^{2^{s}}\left(\theta_{4}\right)=\sum_{k=1}^{4} a(s)_{k} \theta_{k}$. Using the Cartan relations, we now write

$$
\begin{aligned}
\sum_{k=1}^{4} a(s)_{k} \theta_{k} & =S q^{2^{s}} \theta_{4}=\sum_{r=0}^{2^{s}} S q^{r}\left(\sigma^{2^{l}}\right) S q^{2^{s}-r}(\pi) \\
& =\sum_{r=0}^{2^{s}-1}\left(\lambda_{r} \mu_{2^{s}-r} \sigma^{2^{l}} \pi\right)+S q^{2^{s}}\left(\sigma^{2^{l}}\right) \pi .
\end{aligned}
$$

Collecting terms with a factor of $\pi$, we find that

$$
\kappa \pi \stackrel{\text { def }}{=}\left[a(s)_{4} \sigma^{2^{l}}+\sum_{r=0}^{2^{s}-1} \lambda_{r} \mu_{2^{s}-r} \sigma^{2^{l}}+S q^{2^{s}}\left(\sigma^{2^{l}}\right)\right] \pi=\sum_{k=1}^{3} a(s)_{k} \theta_{k},
$$

where $\kappa$ is defined to be the coefficient of $\pi$ in equation (36). Multiplying through by $\sigma^{2^{l}}$, we find that

$$
\kappa \theta_{4}+\sum_{k=1}^{3} a(s)_{k} \sigma^{2^{l}} \theta_{k}=0 .
$$

Equation (37) gives a relation between the $\theta_{j}$ 's whose coefficients are of degree

$$
\operatorname{deg}(\kappa)=2^{s}+\operatorname{deg}\left(\sigma^{2^{l}}\right)=2^{s}+m-\operatorname{deg}(\pi) .
$$

But $\operatorname{deg}(\pi) \geq 2^{l} \cdot 5>2^{l+2} \geq 2^{s}$ by Lemma II.17, and thus we have $\operatorname{deg}(\kappa)<$ $m=\operatorname{deg}(\theta)$. As the $\theta$ 's form an independent set, Fact I.2 implies that the 
coefficients, and in particular $\kappa$, must vanish. Viewing equation (36), in which $\kappa$ was defined, modulo $\sigma^{2^{l}}$, we find that $\sigma^{2^{l}}$ divides $S q^{2^{s}}\left(\sigma^{2^{l}}\right)$. This completes the induction step and proves the claim.

Now for $0 \leq \beta \leq 2$ we have $\sigma^{2^{l}} \mid S q^{2^{l+\beta}}\left(\sigma^{2^{l}}\right)=\left(S q^{2^{\beta}}(\sigma)\right)^{2^{l}}$, the equality holding as in Fact I.8. Taking $\left(2^{l}\right)$ th roots, we find that $\sigma$ divides $S q^{2^{\beta}}(\sigma)$ for $0 \leq \beta \leq 2$. From this it follows that $\sigma$ divides $Q_{\beta}(\sigma)$ for $0 \leq \beta \leq 3$, so that by Corollary II.22, we have that $\sigma=\tau^{2} \pi_{0}$ for some polynomial $\tau$ and some product $\pi_{0}$ of linear factors. Thus $\theta_{4}=\sigma^{2^{l}} \pi=\tau^{2^{i+1}} \pi_{0}^{2^{l}} \pi$. We take $\pi^{\prime}=\pi_{0}^{2^{l}} \pi$ to prove the proposition.

\section{Main theorem, Part II}

In this section, we use Proposition III.8 to complete the proof of the main theorem.

Proposition III.9. Let $H \simeq \mathrm{Z} /(5)$, and let $G=H \ltimes_{\tau} E_{4}$ be a group of type II.

1. If $m$ is an integer not of the form $2^{t} \cdot 5$, then $P(G, 4, m)$ is true.

2. If $m=2^{t} \cdot 5$ for some $t \geq 2$ and $\mathscr{S}=\left\{\theta_{1}, \ldots, \theta_{4}\right\}$ is a 4-element l.s.o.p. of $H^{*}(G)$ in degree $m$, then either $\theta_{j}$ is a square for all $i$, or else the linear span $\mathscr{V}\left\{\theta_{1}, \ldots, \theta_{4}\right\}$ has a basis $\phi_{1}, \ldots, \phi_{d}$ such that

$$
\phi_{j}=\psi_{j}^{2^{t-1}}, \quad 1 \leq j \leq 3 ; \quad \phi_{4}=\psi_{4}^{2^{t-1}} \rho
$$

where $\rho$ is a 2-orbit nonsquare and the elements $\psi_{1}, \ldots, \psi_{3} \in k\left[y_{1}, \ldots, y_{4}\right]_{(10)}^{H}$ satisfy the conditions $S q^{1}\left(\psi_{j}\right)=0$ and $\psi_{j} \mid S q^{2}\left(\psi_{j}\right)$, and generate an ideal invariant under $S q^{4}$.

Proof. Note that by Proposition III.3, the proposition is trivially true if $m$ is not a multiple of 10 . Now fix $m=10 n \geq 20$, and let $\mathscr{S}=\left\{\theta_{1}, \ldots, \theta_{4}\right\}$ be an $\mathscr{A}(2)$-invariant 1.s.o.p. of $\left(k\left[y_{1}, \ldots, y_{4}\right]\right)^{H}$. By Proposition III.6, the linear span $\mathscr{V}\left\{\theta_{1}, \ldots, \theta_{4}\right\}$ has a basis $\theta_{1}^{\prime}, \ldots, \theta_{4}^{\prime}$ such that $\theta_{j}^{\prime}=\phi_{j}^{2}$ for $1 \leq j \leq 3$ and $\theta_{4}^{\prime}=\phi_{4}^{2} \rho$ where $\rho$ is either 1 or a 2-orbit nonsquare. If $\rho=1$, then $\theta_{1}^{\prime}, \ldots, \theta_{4}^{\prime}$ are all squares, and consequently the $\theta_{1}, \ldots, \theta_{4}$ are all squares as well, being linear combinations in characteristic 2 of the $\theta_{j}^{\prime}$ 's.

Suppose then that $\rho$ is a 2-orbit nonsquare. We now apply Proposition III.8 as long as the degree condition is satisfied. If $l$ is defined by

$$
14 \cdot 2^{l-1}<m \leq 14 \cdot 2^{l}
$$

we find that $\mathscr{V}\left\{\theta_{j}, \ldots, \theta_{4}\right\}$ has a basis $\phi_{1}, \ldots, \phi_{d}$ such that $\phi_{j}=\psi_{j}^{2^{l}}$ for $1 \leq j \leq 3$ and $\phi_{4}=\psi_{4}^{2^{l}} \sigma$ for some polynomials $\psi_{j} \in\left(k\left[y_{1}, \ldots, y_{4}\right]\right)^{H}$ and some 2-orbit nonsquare $\sigma$. Now $m=2^{l} \operatorname{deg}\left(\psi_{1}\right)$ is divisible by $2^{l}$, and $m$ is divisible by 5 by Proposition III.3, so we may write $m=2^{l} \cdot 5 c$ for some integer $c$. From equation (38), we have $\frac{7}{5}<c \leq \frac{14}{5}$, so that $c=2$ and $m=2^{l+1} \cdot 5$.

If in fact $m$ is not of the form $2^{t} \cdot 5$, then the above argument shows that to assume that $\rho \neq 1$ leads to a contradiction. Thus $P(G, d, m)$ holds for $m$ not of this form. If, on the other hand, $m=2^{l+1} \cdot 5 \geq 20$, we apply first Lemma III. 7 and then Proposition III. 2 to the situation of the previous paragraph to see that indeed the $\psi_{j}$ have the properties claimed. 
Corollary III.10. Let $G$ be a group of type II, and let $m$ be an integer not of the form $2^{t} \cdot 5$. Then $H^{*}(G) \simeq\left(k\left[y_{1}, \ldots, y_{4}\right]\right)^{H}$ has no $\mathscr{A}(2)$-invariant l.s.o.p.'s in degree $m$.

Proof. Follows in view of Proposition I.14.

Finally, we have

Theorem III.11 (Main Theorem, Part II). Suppose that the group $\mathscr{G}$ contains a subgroup $G \simeq \mathrm{Z} /(5) \ltimes_{\tau} E_{4}$ of type II, and let $m$ be an integer such that $m \neq 2^{t} \cdot 5$ for all $t$. Then $\mathscr{G}$ does not act freely and $k$-cohomologically trivially on any finite complex $X \sim_{2}\left(S^{m-1}\right)^{4}$.

Proof. Immediate from Theorem I.13 and Proposition III.10.

\section{REFERENCES}

[AB88] A. Adem and W. Browder, The free rank of symmetry of $\left(S^{n}\right)^{k}$, Invent Math. 92 (1988), 431-440.

[AW80] J. F. Adams and C. W. Wilkerson, Finite $H$-spaces and algebras over the Steenrod algebra, Ann. of Math. 111 (1980), 95-143.

[Car80] G. Carlsson, On the non-existence of free actions of elementary abelian groups on products of spheres, Amer. J. Math. 102 (1980), 1147-1157.

[Car81] _ Some restrictions on finite groups acting freely on $\left(S^{n}\right)^{k}$, Trans. Amer. Math. Soc. 264 (1981), 449-457.

[CS] H. E. A. Campbell and P. S. Selick, Polynomial algebras over the Steenrod algebra, Comment. Math. Helv. 65 (1990), 171-180.

[Oli78] R. Oliver, Free compact group actions on products of spheres, Proc. Aarhus Sympos. on Algebraic Topology, Springer-Verlag, 1978, pp. 539-548.

[Ser65a] J.-P. Serre, Algèbre locale. Multiplicités, Springer-Verlag, 1965.

[Ser65b] __, Sur la dimension cohomologique des groupes finis, Topology 3 (1965), 412-420.

[Woo86] R. M. W. Wood, Splitting $\Sigma\left(\mathbf{C} P^{\infty} \times \cdots \times \mathbf{C} P^{\infty}\right)$ and the action of Steenrod squares $S q^{i}$ on the polynomial ring $\mathbf{F}_{2}\left[x_{1}, \ldots, x_{n}\right]$, Algebraic Topology, Barcelona, Lecture Notes in Math., Vol. 1298, Springer, Berlin and New York, 1986, pp. 237-255.

Department of Mathematics, University of Michigan, Ann Arbor, Michigan 48109

E-mail address: judith.silverman@math.lsa.umich.edu 